\title{
Minimum Strictly Convex Quadrangulations of Convex Polygons
}

\author{
Matthias Müller-Hannemann \\ Karsten Weihe
}

Konstanzer Schriften in Mathematik und Informatik

Nr. 13, Juli 1996

ISSN 1430-3558

(C) Fakultät für Mathematik und Informatik Universität Konstanz

Postfach 5560/D 188, 78434 Konstanz, Germany

Email: preprints@informatik.uni-konstanz.de

WWW: http://www.informatik.uni-konstanz.de/Schriften 


\title{
Minimum Strictly Convex Quadrangulations of Convex Polygons
}

\author{
Matthias Müller-Hannemann ${ }^{1} \quad$ Karsten Weihe $^{2}$ \\ $13 / 1996$ \\ Universität Konstanz \\ Fakultät für Mathematik und Informatik
}

\begin{abstract}
We present a linear-time algorithm that decomposes a convex polygon conformally into a minimum number of strictly convex quadrilaterals. Moreover, we characterize the polygons that can be decomposed without additional vertices inside the polygon, and we present a linear-time algorithm for such decompositions, too. As an application, we consider the problem of constructing a minimum conformal refinement of a mesh in the three-dimensional space, which approximates the surface of a workpiece. The latter problem has resulted from a cooperation with an engineering company that sells CAD packages. We have proved that this problem is strongly $\mathcal{N} \mathcal{P}$-hard, and we present a linear-time algorithm with a constant approximation ratio of 4 .
\end{abstract}

\section{Introduction}

In this paper, we introduce a new approach to the decomposition of convex polygons and, as an application, to the conformal refinement of finite element meshes into strictly convex quadrilaterals.

Much work has been done on decompositions

\footnotetext{
${ }^{1}$ Technische Universität Berlin, Sekr. MA 6-1, Str.d. 17.Juni 136, 10623 Berlin, Germany, mhannema@math.tuberlin.de, http://winnie.math.tu-berlin.de/ ${ }^{\sim}$ mhannema.

This author was supported by the special program "Efficient Algorithms for Discrete Problems and Their Applications" of the Deutsche Forschungsgemeinschaft under grant Mo 446/2-2.

${ }^{2}$ Universität Konstanz, Fakultät für Mathematik und Informatik, Postfach 5560/D188, 78434 Konstanz, Germany, karsten.weihe@uni-konstanz.de, http://www.informatik.uni-konstanz.de/ ${ }^{\sim}$ weihe.
}

into triangles; see [4] and [1] for surveys. However, there is much less work on quadrilaterals, although quadrilaterals are more appropriate in certain situations. Ref. [5] states one possible reason for that: "good quadrilateral meshes are harder to generate than good triangular meshes." See [12] for a survey of the work on related topics.

Conformal decompositions of polygons. A polygon is a closed sequence of straight lines (edges) in the plane or, more generally, of smooth curves in a smooth surface in the threedimensional space. A polygon is simple if it does not cross itself, and convex if the internal angle at each vertex is at most $\pi$. A vertex is a corner if its internal angle is strictly less than $\pi$. A polygon is strictly convex if all its vertices are corners.

Throughout this paper, a conformal decomposition of a simple polygon is a decomposition of its enclosed area into strictly convex quadrilaterals such that the following holds (see Fig. 1):

1. The quadrilaterals are openly disjoint.

2. Each edge of the polygon is an edge of exactly one quadrilateral. In particular, additional vertices on the boundary of the polygon are not allowed (nonetheless in the interior).

3. If two quadrilaterals share more than one corner, they share exactly one edge as a whole.

There is a conformal decomposition for a polygon $P$ if and only if the number of vertices of $P$ is even (cf. Lemma 2.2). This fact will be proved only for convex polygons, because this is our focus in this work, but the proof can be easily extended to general simple polygons.

We call a conformal decomposition of a convex polygon $P$ perfect if it has no internal vertices. In 


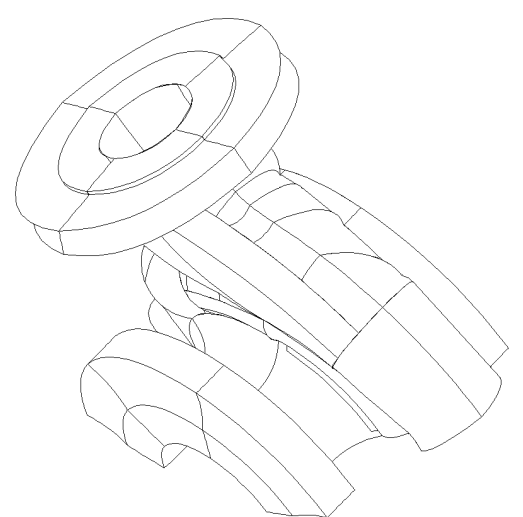

Figure 2: a mesh modeling a pump. This mesh has been constructed by a German car company.

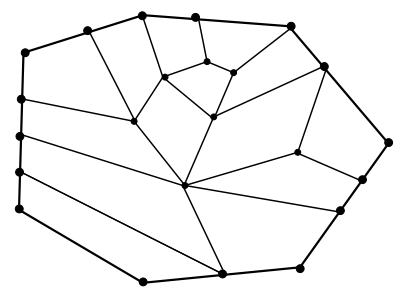

Figure 1: A convex polygon with 7 corners and 16 vertices and a conformal decomposition with 7 additional, internal vertices.

other words, all internal edges are chords of $P$. If $P$ admits a perfect conformal decomposition, we call $P$ perfect, too. In Sect. 2 , we will prove a useful characterization of perfect polygons (Lemmas 2.6 and 2.8). Based on this result, we will present a linear-time algorithm that tests whether a given polygon $P$ is perfect and, if so, constructs a perfect decomposition.

In Sect. 3, we will present a linear-time algorithm that constructs a conformal decomposition of a convex polygon with a minimum number of quadrilaterals. This problem is closely related to perfect decompositions, since for perfect polygons, the perfect decompositions are exactly the minimum decompositions (cf. Corollary 3.3).

To our knowledge, neither problem has been dealt with so far. Nonetheless, a few related problems have found some attention. Ref. [2] gives lower and upper bounds on the number of quadrilaterals in a conformal decomposition of simple, not necessarily convex polygons (even with holes),

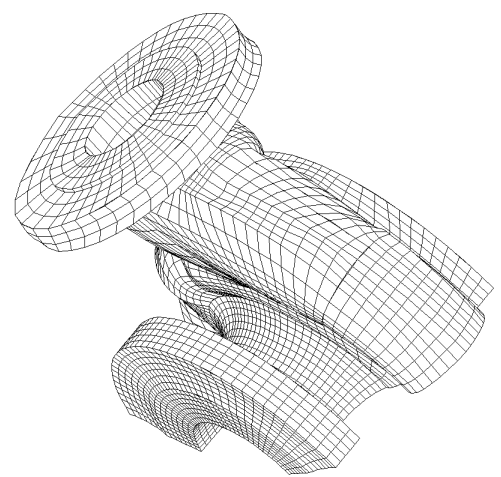

Figure 3: The conformal refinement produced by the heuristic in [7]. Note that the decomposition of an original polygon is usually not conformal.

but in contrast they also allow additional vertices to be placed on the boundary. Refs. $[9,10]$ investigate perfect decompositions of (star-shaped) rectilinear polygons into non-strictly convex quadrilaterals, and [6] considers perfect decompositions of non-convex polygons but even allows overlapping internal edges. See [12] for a systematic survey.

Conformal mesh refinement. Section 4 is devoted to an application, which has motivated the research on decompositions of quadrilaterals: Conformal refinements of finite-element meshes are important for the computer aided design of machines, vehicles, and many other kinds of technical devices. Such a mesh approximates the surface of a workpiece; see Figs. 2 and 3. This procedure prepares an interactively generated model of the workpiece for numerical computations.

A mesh is a complex of openly disjoint, convex polygons. In a conformal refinement of a mesh, each polygon is decomposed into strictly convex quadrilaterals, but not necessarily conformally. Instead, the common decomposition of all polygons fulfills the first and third conditions of conformality; see Fig. 3. The solution in Fig. 3 is constructed by a heuristic, which is based on bidirected network flows [7].

In [7] and all other work we know of, the number of quadrilaterals is not considered explicitly, although this number is crucial for the run time of all later steps of the CAD process. For this 
reason, the problem we consider in this paper is the following:

Find a conformal refinement with a minimum total number of quadrilaterals (minimum conformal mesh refinement problem).

This problem definition might not reflect all practical aspects, but allows better insights into the mathematics of the problem. In Sect. 4 we discuss the problem further and present the following results:

- The minimum conformal mesh refinement problem is $\mathcal{N} \mathcal{P}$-hard.

- There is a linear-time approximation algorithm which yields an approximation ratio of 4.

This algorithm relies heavily on the results about decompositions of polygons in Sects. 2 and 3.

\section{Perfect Polygons}

In this section, we sketch the proof of the following theorem.

Theorem 2.1 There is a linear-time algorithm which tests whether or not a polygon is perfect and, if so, constructs a perfect conformal decomposition.

To prove Theorem 2.1, we will need some general notation. An interval on a polygon $P$ is a path on its boundary. An interval is trivial if it consists of exactly one edge of $P$. A segment $S$ is an interval between two successive corners of $P$. Let $e_{1}$ and $e_{2}$ be two different edges of $P$. Then $I\left[e_{1}, e_{2}\right]$ denotes the interval counterclockwise from $e_{1}$ to $e_{2}$, including neither $e_{1}$ nor $e_{2}$.

A conformal decomposition of $P$ is usually identified with the planar, embedded graph $G=$ $(V, E)$ whose outer face is $P$ and whose internal faces are the quadrilaterals. For such a graph $G=(V, E)$, the dual graph $G^{d}=\left(V^{d}, E^{d}\right)$ is defined as usual [8]: $V^{d}$ is the set of all faces of $G$, and two vertices in $V^{d}$ are adjacent if and only if they are incident to a common edge of $G$. Let $G^{*}=\left(V^{*}, E^{*}\right)$ be the graph arising from $G^{d}$ by removing the vertex corresponding to the outer face of $G$. See Fig. 4. A planar graph whose vertices all lie on a common face is called outerplanar.
Hence, a perfect decomposition corresponds to an outerplanar graph.

Lemma 2.2 [5] $A$ convex polygon $P$ admits a conformal decomposition if and only if the number of vertices of $P$ is even.

For completeness, we sketch an illustrative proof.

Proof: The if part follows from the construction in Fig. 5.

Fig. 4 demonstrates an obvious fact: that the degree of a face $v^{*} \in E^{*}$ has in $G^{*}$ the same parity as the number of edges of $P$ incident to $v^{*}$. Now the only-if part follows from the fact that the sum of all degrees in an undirected graph $G^{*}$ is always even.

Assumption 2.3 Throughout Sects. 2 and 3, we assume that the number of vertices of $P$ is even.

Note that $G^{*}$ is always connected. The following observation is well-known for outerplanar graphs and is also obvious from Fig. 4.

Observation 2.4 $G$ is a perfect decomposition if and only if $G^{*}$ is a tree.

Each degree-one vertex $v^{*}$ of $G^{*}$ points to a trivial segment of $P$ (see Fig. 4). In the quadrilateral corresponding to $v^{*}$, this is the edge opposite to the edge crossed by the unique incident dual edge. We will sometimes identify such a vertex with this trivial segment. Since each tree has at least two leaves, we obtain:

Corollary 2.5 Every perfect polygon contains at least two trivial segments.

The length $L(I)$ of an interval $I$ is the number of its edges. Moreover, $K(I)$ denotes the maximum size of a choice of strictly convex internal vertices of $I$ such that no two of them are neighbored on $P$. We often denote $(L-2 K)(I):=L(I)-2 \cdot K(I)$. Note that $(L-2 K)(I)$ is always nonnegative.

Roughly speaking, Lemmas 2.6 and 2.8 show that, if a polygon is perfect, it even admits a perfect decomposition of a very restricted shape: The reduced dual graph $G^{*}$ is either a path, or it has a single branching vertex, which has degree three. 


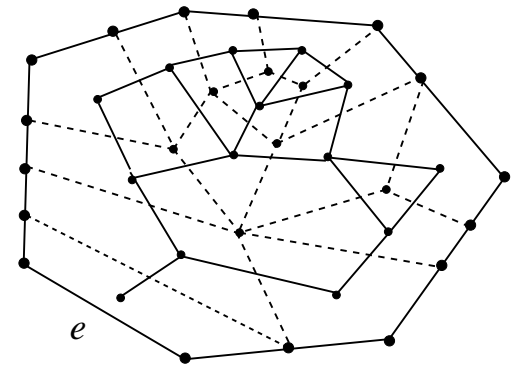

Figure 4: The polygon $P$ (solid) and the conformal decomposition $G$ (dashed) of Fig. 1, and the graph $G^{*}$ (solid). The unique degree-one vertex of $G^{*}$ points to $e$.

In the former case, it is called a path decomposition, and in the latter case, a $K_{1,3}-$ decomposition, because then $G^{*}$ is a subdivision of the complete bipartite graph $K_{1,3}$ on $1+3$ vertices.

Lemma 2.6 Let $P$ be a polygon with exactly two trivial segments $e_{1}$ and $e_{2}$, and let $I_{1}:=I\left[e_{1}, e_{2}\right]$ and $I_{2}:=I\left[e_{2}, e_{1}\right]$. W.l.o.g. we have $L\left(I_{1}\right) \geq$ $L\left(I_{2}\right)$. Then $P$ is perfectly decomposable if and only if $(L-2 K)\left(I_{1}\right) \leq L\left(I_{2}\right)$. In this case, it admits a path decomposition.

Proof: As each tree with two leaves is a path, any perfect decomposition is a path decomposition. When $G^{*}$ is a path, each vertex of $G^{*}$ corresponds to a quadrilateral that shares exactly two edges with $I_{1} \cup I_{2}$. If these two edges are on the same interval $I_{1}$ or $I_{2}$, they are incident and enclose a corner of $P$. (Otherwise, they would enclose a third leaf of $G^{*}$.) Therefore, we have $L\left(I_{1}\right)-L\left(I_{2}\right) \leq$ $2 \cdot K\left(I_{1}\right)$ and $L\left(I_{2}\right)-L\left(I_{1}\right) \leq 2 \cdot K\left(I_{2}\right)$. Obviously, these two inequalities are also sufficient for perfectness. The latter inequality is always true because of $L\left(I_{1}\right) \geq L\left(I_{2}\right) \geq(L-2 K)\left(I_{2}\right)$. Hence, the former inequality determines perfect decomposability, which proves Lemma 2.6.

In Lemma 2.8, we assume the following scenario.

Scenario 2.7 Let $P$ be a polygon with at least three trivial segments. Let $e_{1}, e_{2}$, and $e_{3}$ be three trivial segments such that the counterclockwise order around $P$ is $e_{1} \prec e_{2} \prec e_{3} \prec e_{1}$. Let

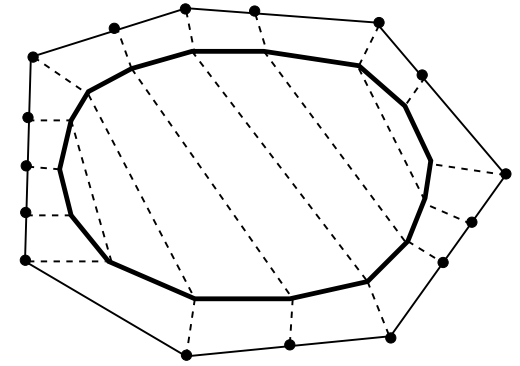

Figure 5: proof of Lemma 2.2. First the convex polygon is reduced to a strictly convex core (thick solid line), then the core can be refined in a straightforward manner. Obviously, this procedure does not minimize the number of quadrilaterals in general.

$I_{1}:=I\left[e_{1}, e_{2}\right], I_{2}:=I\left[e_{2}, e_{3}\right], I_{3}:=I\left[e_{3}, e_{1}\right]$, and w.l.o.g. $L\left(I_{1}\right) \geq L\left(I_{2}\right)$ and $L\left(I_{1}\right) \geq L\left(I_{3}\right)$. Assume that $L\left(I_{1}\right)$ is minimum subject to all these conditions.

Fig. 6 shows an example for this scenario.

Lemma 2.8 In Scenario 2.7, $P$ is perfectly decomposable if and only if $(L-2 K)\left(I_{1}\right) \leq L\left(I_{2}\right)+$ $L\left(I_{3}\right)+1$. In this case, there is a perfect decomposition that is either a path decomposition with leaves $e_{1}$ and $e_{2}$, or it is a $K_{1,3}$-decomposition with leaves $e_{1}, e_{2}$, and $e_{3}$.

Proof: We consider four different cases separately.

Case $I:(L-2 K)\left(I_{1}\right)=L\left(I_{2}\right)+L\left(I_{3}\right)+1$.

Then $L\left(I_{1}\right) \geq L\left(I_{2}\right)+L\left(I_{3}\right)+1=L\left(I_{2} \cup e_{3} \cup I_{3}\right)$, and the existence of a path $G^{*}$ with leaves $e_{1}$ and $e_{2}$ follows analogously to Lemma 2.6.

Case II: $L\left(I_{2}\right)=0$ or $L\left(I_{3}\right)=0$.

W.l.o.g., let $L\left(I_{2}\right)=0$. By Assumption 2.3, $L\left(I_{1}\right)+L\left(I_{2}\right)+L\left(I_{3}\right)$ is odd. Hence, $L\left(I_{1}\right)$ and $L\left(I_{3}\right)$ have different parities, and the overall assumption $L\left(I_{1}\right) \geq L\left(I_{3}\right)$ can be strengthened to $L\left(I_{1}\right) \geq L\left(I_{3}\right)+1=L\left(I_{2} \cup e_{3} \cup I_{3}\right)$. Again the existence of a path $G^{*}$ with leaves $e_{1}$ and $e_{2}$ follows analogously to Lemma 2.6 .

Case III: $(L-2 K)\left(I_{1}\right)<L\left(I_{2}\right)+L\left(I_{3}\right)+1$ and $L\left(I_{2}\right)>0$ and $L\left(I_{3}\right)>0$. 
This is the case shown in Fig. 6. We first define three values $b_{1}, b_{2}$, and $b_{3}$. Recall the overall assumption $L\left(I_{1}\right) \geq L\left(I_{2}\right)$ and $L\left(I_{1}\right) \geq L\left(I_{3}\right)$. In particular, this yields $L\left(I_{1}\right) \geq\left|L\left(I_{2}\right)-L\left(I_{3}\right)\right|$. Together with the condition defining Case III, we obtain that the interval $\mathcal{I}:=$

$$
\left[(L-2 K)\left(I_{1}\right), L\left(I_{1}\right)\right] \cap\left[\left|L\left(I_{2}\right)-L\left(I_{3}\right)\right|, L\left(I_{2}\right)+L\left(I_{3}\right)\right]
$$

is not empty. If $|\mathcal{I}|=1$, the unique element is either $(L-2 K)\left(I_{1}\right)$ or $L\left(I_{1}\right)$, because $L\left(I_{2}\right), L\left(I_{3}\right)>$ 0 implies $\left|\left[\left|L\left(I_{2}\right)-L\left(I_{3}\right)\right|, L\left(I_{2}\right)+L\left(I_{3}\right)\right]\right|>1$. In this case, we define $b_{1}$ as this unique element of $\mathcal{I}$, and hence $b_{1}+L\left(I_{2}\right)+L\left(I_{3}\right)$ is odd. On the other hand, if $|\mathcal{I}|>1$, we choose $b_{1} \in \mathcal{I}$ so that $b_{1}+L\left(I_{2}\right)+L\left(I_{3}\right)$ is odd again. Next define $b_{2}:=L\left(I_{2}\right)-1$ and $b_{3}:=L\left(I_{3}\right)$ and consider the following linear equation system:

$$
\left(\begin{array}{ccc|c}
0 & 1 & 1 & b_{1} \\
1 & 0 & 1 & b_{2} \\
1 & 1 & 0 & b_{3}
\end{array}\right)
$$

The unique solution is

$$
\begin{aligned}
& x_{1}=\left(b_{2}+b_{3}-b_{1}\right) / 2, \\
& x_{2}=\left(b_{1}+b_{3}-b_{2}\right) / 2, \\
& x_{3}=\left(b_{1}+b_{2}-b_{3}\right) / 2 .
\end{aligned}
$$

By definition of the $b_{i}, b_{1}+b_{2}+b_{3}$ is even. Hence, the $x_{i}$ are integral. Next we show that the $x_{i}$ are nonnegative. Because of $b_{1} \in \mathcal{I}$, we have $b_{1} \leq L\left(I_{2}\right)+L\left(I_{3}\right)$, and since $b_{1}$ and $L\left(I_{2}\right)+L\left(I_{3}\right)$ have different parities, we even have $b_{1} \leq b_{2}+b_{3}$, which means $x_{1} \geq 0$. Moreover, $b_{1} \in \mathcal{I}$ immediately yields $b_{1} \geq\left|L\left(I_{2}\right)-L\left(I_{3}\right)\right|$, and by the same parity argument, we even have $b_{1} \geq\left|b_{2}-b_{3}\right|$. This implies $x_{2} \geq 0$ and $x_{3} \geq 0$.

All these facts together suffice, because we can construct a subdivision of $K_{1,3}$ from the values $x_{i}$ (cf. Fig. 6): $x_{1}$ is the number of quadrilaterals sharing one edge with $I_{2}$ and one with $I_{3}$. Analogously, $x_{2}$ quadrilaterals share an edge with $I_{1}$ and $I_{3}$, and $x_{3}$ with $I_{1}$ and $I_{2}$.

There are as many as $\frac{1}{2}\left(L\left(I_{1}\right)-\left(x_{2}+x_{3}\right)\right)$ quadrilaterals that share two edges with $I_{1}$. Note that the number of quadrilaterals that share two edges with $I_{1}$ is geometrically feasible, because we obtain $\frac{1}{2}\left(L\left(I_{1}\right)-\left(x_{2}+x_{3}\right)\right) \leq K\left(I_{1}\right)$ from $(L-2 K)\left(I_{1}\right) \leq b_{1}=x_{2}+x_{3}$.

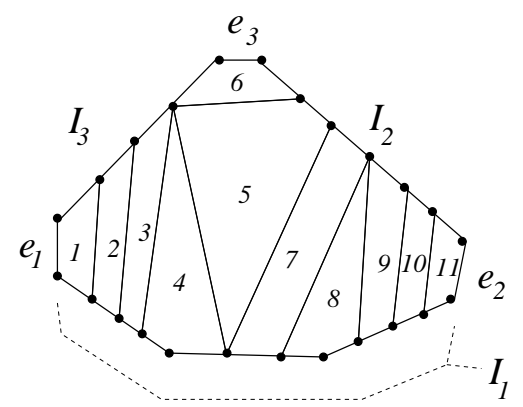

Figure 6: an example for Scenario 2.7. Here we have $L\left(I_{1}\right)=11,(L-2 K)\left(I_{1}\right)=7, L\left(I_{2}\right)=6$, $L\left(I_{3}\right)=4$, and $\mathcal{I}=[7,11] \cap[2,10]=[7,10]$. For this decomposition, we have chosen $b_{1}=7$, $b_{2}=5$, and $b_{3}=4$. This yields $x_{1}=1$ (inducing quadr. no. 6), $x_{2}=3$ (nos. $\left.1,2,3\right)$, and $x_{3}=4$ (nos. $7,9,10,11$ ).

As $b_{2}=L\left(I_{2}\right)-1$, there is one quadrilateral that shares one edge with $I_{2}$ and no edge with $P \backslash I_{2}$. This is the branching vertex of the subdivision of $K_{1,3}$ (no. 5 in Fig. 6 ).

Case IV: $(L-2 K)\left(I_{1}\right)>L\left(I_{2}\right)+L\left(I_{3}\right)+1$.

First we show that $I_{1}$ contains no trivial segment. To see this, assume $I_{1}$ does contain a trivial segment $e_{4}$. Let $I_{4}:=I\left[e_{1}, e_{4}\right]$ and $I_{5}:=I\left[e_{4}, e_{2}\right]$. Clearly, we have $L\left(I_{4}\right)<L\left(I_{1}\right)$ and $L\left(I_{5}\right)<L\left(I_{1}\right)$. In Case IV, we also have $L\left(I_{2} \cup e_{3} \cup I_{3}\right)<L\left(I_{1}\right)$. However, this contradicts the specific choice of $\left\{e_{1}, e_{2}, e_{3}\right\}$ in the scenario of Lemma 2.8, because $\left\{e_{1}, e_{4}, e_{2}\right\}$ would be a strictly better choice.

Now we know that $I_{1}$ contains no trivial segment. Suppose that nonetheless $P$ admits a perfect decomposition $G=(V, E)$ of $P$. Then all leaves of $G^{*}$ belong to $e_{2} \cup I_{2} \cup e_{3} \cup I_{3} \cup e_{1}$. In other words, the quadrilaterals incident to $I_{1}$ form one single path $p$ of $G^{*}$.

Let $Q_{1}$ denote the quadrilaterals sharing one edge with $I_{1}$ and one or more edges with $I_{2} \cup e_{3} \cup$ $I_{3}$, let $Q_{2}$ be the quadrilaterals sharing exactly one edge with $I_{1}$ and none with $I_{2} \cup e_{3} \cup I_{3}$, and let $Q_{3}$ be the quadrilaterals sharing at least one edge with $I_{2} \cup e_{3} \cup I_{3}$ and none with $I_{1}$. Since $I_{1}$ contains no trivial segments, no quadrilateral shares more than two edges with $I_{1}$. Note that at most $K\left(I_{1}\right)$ quadrilaterals share exactly two edges with $I_{1}$. Therefore, we have $\left|Q_{1}\right|+\left|Q_{2}\right| \geq$ $(L-2 K)\left(I_{1}\right)$. 
Next note that each quadrilateral in $Q_{2}$ is a branching vertex of the tree $G^{*}$. The branch that does not belong to $p$ contains at least one leaf, and this leaf belongs to $Q_{3}$. This implies $\left|Q_{3}\right| \geq\left|Q_{2}\right|$. Clearly, we have $\left|Q_{1}\right|+\left|Q_{3}\right| \leq L\left(I_{2}\right)+L\left(I_{3}\right)+$ 1. Altogether, we obtain the contradiction $(L-$ $2 K)\left(I_{1}\right) \leq\left|Q_{1}\right|+\left|Q_{2}\right| \leq\left|Q_{1}\right|+\left|Q_{3}\right| \leq L\left(I_{2}\right)+$ $L\left(I_{3}\right)+1$.

Now we are going to introduce the linear-time algorithm for perfect convex polygons. Recall from Corollary 2.5 that polygons with less than two trivial segments are not perfect. Lemma 2.6 immediately translates into a linear-time algorithm for polygons with exactly two trivial segments.

So consider the case that $P$ has more than two trivial segments. Clearly, the only problem is to find three trivial segments $e_{1}, e_{2}$ and $e_{3}$ as described in Scenario 2.7, because the proof of Lemma 2.8, Cases I-III, immediately yields a linear-time algorithm for the rest.

To find suitable trivial segments $e_{1}, e_{2}, e_{3}$, we apply the following strategy. Let $n$ denote the number of edges of $P$. We number all edges counterclockwise $0 \ldots n-1$, starting anywhere. In the following, all additions are performed modulo $n$. In particular, a subtraction $x-y$ denotes the counterclockwise distance from $y$ to $x$ around $P$.

For a real number $x \in[0, n], \ell(x) \in\{0, \ldots, n-$ $1\}$ denotes the first value $i$ in the sequence $(\lfloor x\rfloor,\lfloor x\rfloor-1,\lfloor x\rfloor-2, \ldots)$ such that the edge no. $i$ is a trivial segment. Analogously, $r(x) \in$ $\{0, \ldots, n-1\}$ denotes the first value $i$ in the sequence $(\lceil x\rceil,\lceil x\rceil+1,\lceil x\rceil+2, \ldots)$ such that the edge no. $i$ is a trivial segment. Clearly, all these values can be computed in linear time during one single pass around $P$.

For an $i \in\{0, \ldots, n-1\}$ that is the number of a trivial segment, let $M(i)$ denote the minimum possible value of $\max \left\{i^{\prime}-i, i^{\prime \prime}-i^{\prime}, i-i^{\prime \prime}\right\}$ such that $i^{\prime}$ and $i^{\prime \prime}$ are numbers of trivial segments and $i<i^{\prime}<i^{\prime \prime}<i$ is the cyclic order modulo $n$. It suffices to compute $M(i)$ for each $i$ and to take the overall minimum. For real numbers $x, y \in[0, n]$ let

$$
A(x, y):= \begin{cases}\frac{x+y}{2}, & x \leq y \\ \frac{x+y}{2}+\frac{n}{2}, & x>y\end{cases}
$$

Intuitively, $A(x, y)$ is the middle of the interval of $P$ that goes counterclockwise from $x$ to $y$. The following lemma fills in the remaining gap.

Lemma 2.9 For $i \in\{0, \ldots, n-1\}, M(i)$ is attained by the following choices of $i^{\prime}$ and $i^{\prime \prime}$.

1. If all trivial segments except $i$ belong to $[i+$ $\left.1,\left\lfloor i+\frac{1}{3} n\right\rfloor\right]$, we choose $i^{\prime \prime}=\ell\left(i+\frac{1}{3} n\right)$, and $i^{\prime}$ is an arbitrary trivial segment different from $i$ and $i^{\prime \prime}$

2. Analogously, if all trivial segments except $i$ belong to $\left[\left\lceil i-\frac{1}{3} n\right\rceil, i-1\right]$, we choose $i^{\prime}=r(i-$ $\left.\frac{1}{3} n\right)$ and $i^{\prime \prime}$ arbitrarily.

3. Otherwise, one of the following choices is appropriate:

\begin{tabular}{c|c}
$i^{\prime}$ & $i^{\prime \prime}$ \\
\hline$\ell\left(i+\frac{1}{3} n\right)$ & $\ell\left(A\left(i^{\prime}, i\right)\right)$ or $r\left(A\left(i^{\prime}, i\right)\right)$ \\
$\ell\left(A\left(i, i^{\prime \prime}\right)\right)$ or $r\left(A\left(i, i^{\prime \prime}\right)\right)$ & $r\left(i-\frac{1}{3} n\right)$ \\
$r\left(i+\frac{1}{3} n\right)$ & $\ell\left(i-\frac{1}{3} n\right)$
\end{tabular}

Proof: In the first case of Lemma 2.9, we have $i^{\prime}-i \leq n / 3, i^{\prime \prime}-i^{\prime} \leq n / 3$, and $i-i^{\prime \prime} \geq 2 n / 3$ for any choice of $i^{\prime}$ and $i^{\prime \prime}$. Therefore, $i^{\prime \prime}$ is best chosen counterclockwise "farthest away" from $i$, which means $i^{\prime \prime}=\ell\left(i+\frac{1}{3} n\right)$. Clearly, $i^{\prime}$ may be chosen arbitrarily. The second case is mirror-symmetric.

In the third case, we apply a further case distinction. For $i^{\prime} \in\left[i+1,\left\lfloor i+\frac{1}{3} n\right\rfloor\right]$, we have $i^{\prime}-i \leq \max \left\{i^{\prime \prime}-i^{\prime}, i-i^{\prime \prime}\right\}$, because $i^{\prime}-i \leq \frac{1}{3} n$ and all three differences sum up to $n$. Hence, the best choice for $i^{\prime}$ is $i^{\prime}=\ell\left(i+\frac{1}{3} n\right)$. To minimize $\max \left\{i^{\prime \prime}-i^{\prime}, i-i^{\prime \prime}\right\}$, we clearly choose $i^{\prime \prime}$ as close to $A\left(i^{\prime}, i\right)$ as possible, that is, $i^{\prime \prime}=\ell\left(A\left(i^{\prime}, i\right)\right)$ or $i^{\prime \prime}=r\left(A\left(i^{\prime}, i\right)\right)$. The case $i^{\prime \prime} \in\left[\left\lceil i-\frac{1}{3} n\right\rceil, i-1\right]$ is mirror-symmetric.

It remains the case $i^{\prime}, i^{\prime \prime} \in\left[i+\frac{1}{3} n, i-\frac{1}{3} n\right]$ (note that $\left.i-\frac{1}{3} n=i+\frac{2}{3} n\right)$. Then we have $i^{\prime \prime}-i^{\prime} \leq \frac{1}{3} n$, $i^{\prime}-i \geq \frac{1}{3} n$, and $i-i^{\prime \prime} \geq \frac{1}{3} n$, which means that $i^{\prime}$ and $i$ " should be chosen "farthest away" from each other, that is, $i^{\prime}=r\left(i+\frac{1}{3} n\right)$ and $i^{\prime \prime}=\ell\left(i-\frac{1}{3} n\right)$. 


\section{Non-Perfect Polygons}

Remember Assumption 2.3. For a conformal decomposition $G=(V, E)$ of polygon $P$ let $q(G)$ denote the number of internal, quadrangular faces. In this section, we will sketch the proof of the following result.

Theorem 3.1 There is a linear-time algorithm that constructs, for a convex polygon, a conformal decomposition $G$ that minimizes $q(G)$.

Let $i(G)$ denote the number of internal vertices, that is, the members of $V$ that do not lie on $P$. In particular, a decomposition is perfect if and only if $i(G)=0$. The following lemma relates $i(G)$ to $q(G)$.

Lemma 3.2 For a fixed polygon $P, q(G)$ grows strictly monotonously with $i(G)$. Therefore, to minimize $q(G)$ it suffices to minimize $i(G)$.

Proof: Euler's formula says $|V|+(q(G)+1)=$ $|E|+2$. Let denote $\left(V_{1}, E_{1}\right)=P$, and let $E_{2}:=E \backslash$ $E_{1}$ denote the set of all internal edges of $G$. Then we have $\left|V_{1}\right|=\left|E_{1}\right|$, and since all internal faces of $G$ are quadrilaterals, we also have $4 \cdot q(G)=$ $\left|E_{1}\right|+2 \cdot\left|E_{2}\right|$. Together, this yields $q(G)=i(G)+$ $\left|E_{1}\right| / 2-1$.

Corollary 3.3 If existing, a perfect decomposition $G$ of a polygon $P$ minimizes $q(G)$.

Therefore, the algorithm from Sect. 2 solves the problem for perfect polygons. For non-perfect polygons, we abstract from all geometrical aspects and restrict our attention to purely structural aspects. More precisely, in Lemma 3.4 we first characterize the planar graphs induced by conformal decompositions.

Lemma 3.4 Let $P$ be a convex polygon and $G=$ $(V, E)$ a biconnected planar graph together with a designated face $F$ such that

1. $F$ is isomorphic to $P$;

2. all other faces of $G$ are quadrilaterals;

3. the following vertices have degree at least 3: all internal vertices and all vertices on $F$ that do not correspond to corners of $P$;

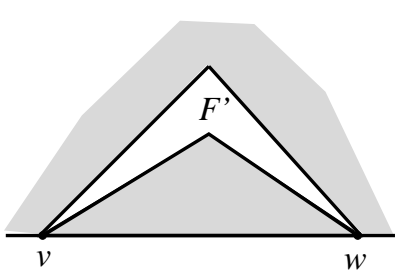

(a)

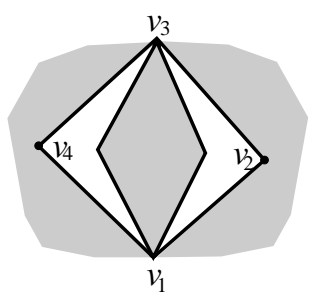

(b)
Figure 7: The schematic situations prevented by the fourth and fifth condition in Lemma 3.4, respectively.

4. if $F$ shares two vertices $v, w \in G$ with another face $F^{\prime}$ such that $v$ and $w$ correspond to vertices on the same segment of $P$, then $v$ and $w$ are adjacent in $G$ (see Fig. 7(a) for the situations prevented by this condition);

5. when two non-adjacent vertices, $v_{1}$ and $v_{3}$, on a cycle $\left(v_{1}-v_{2}-v_{3}-v_{4}-v_{1}\right)$ of length 4 are removed, each connected component of $G \backslash$ $\left\{v_{1}, v_{3}\right\}$ contains $v_{2}$ or $v_{4}$ (see Fig. 7(b) for the situations prevented by this condition).

Then (and only then) there is a planar embedding of $G$ such that the outer face is mapped onto $P$ and all internal faces are strictly convex.

Proof: Omitted (see Appendix A).

It is easy to see that all these preconditions are necessary. Our proof of sufficiency is constructive and yields a linear-time algorithm for a geometrically feasible embedding of such a graph $G$ (all details omitted for brevity). Hence, from now on we may restrict our attention to the structural aspects of $G$. It is also easy to see from Fig. 7 that the last two conditions of Lemma 3.4 are fulfilled for each optimal decomposition. Thus, we may even restrict our attention to the first three conditions. These conditions will be trivially fulfilled by our construction.

Lemma 3.5 below is the basis of the linear-time algorithm for non-perfect polygons. To state this lemma, we need some more terminology.

We will denote a polygon by the counterclockwise sequence of the lengths of its segments. For example, $(1,1,1,1)$ denotes the strictly convex quadrilateral, $(1,1,2)=(1,2,1)=(2,1,1)$ the quadrilateral degenerated to a triangle, and 


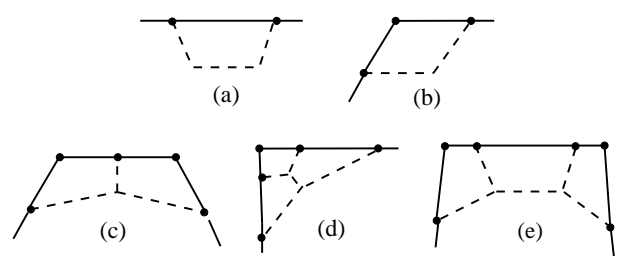

Figure 8: The first class of cut components in Lemma 3.5(2). The solid lines belong to $P$, and the dashed lines are internal edges. By Lemma 3.4, only the structure of the graph matters; the concrete lengths and angles are only exemplary.

$(4,1,2,3,2,2,2)=(1,2,3,2,2,2,4)=\cdots$ the polygon in Fig. 1. By Lemma 3.4, this notation encodes all information that we need for our purpose.

Recall the definition of $G^{*}=\left(V^{*}, E^{*}\right)$ from the beginning of Sect. 2. Let $G=(V, E)$ be an undirected planar, embedded graph. An area component of $G$ is a subgraph $G^{\prime}$ induced by a connected component of $G^{*}$. More precisely, $G^{\prime}$ consists of all vertices and edges incident to the polygons that correspond to this component of $G^{*}$. An area decomposition of $G$ is a collection of area components such that the inducing components of $G^{*}$ partition all vertices in $V^{*}$. Intuitively, this means that the internal faces of $G$ are partitioned and covered by closed, but openly disjoint, connected areas.

Lemma 3.5 For $P \notin\{(2,1,1),(3,3,3,3)$, $(4,3,3),(4,2,2)\}$, there is a conformal decomposition $G$ with minimum $q(G)$ such that there is an area decomposition of $G$ with the following properties:

1. The area decomposition consists of at most four area components.

2. All area components except one are isomorphic to one of the components depicted in Figs. 8 and 9. These area components are henceforth called the cut components.

3. The remaining area component is outerplanar. This area component is henceforth called the core component.

4. No two cut components share an edge.

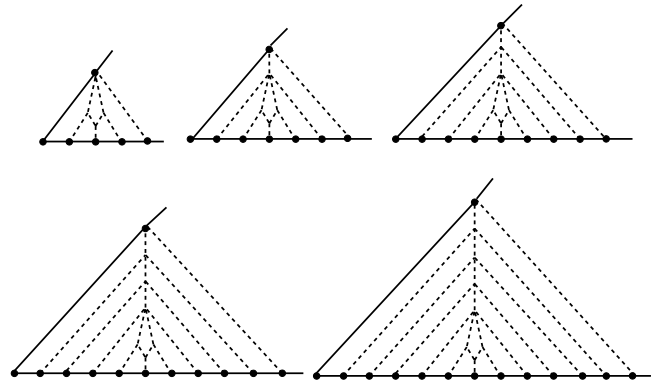

Figure 9: The five smallest cut components of the second class. The definition of the whole (infinite) class might be obvious.

5. All cut components except at most one are of type (c), (d), or (e) in Fig. 8.

6. If a cut component of type (a) or (b) in Fig. 8 or a cut component in Fig. 9 occurs, the core component admits a path decomposition.

7. If a cut component of the type in Fig. 9 occurs, the area decomposition contains at most two cut components; if a cut component of type (a) or (b) in Fig. 8 occurs, this is the only cut component.

Proof: Omitted (see Appendix B).

In the following, we will only sketch the lineartime algorithm that performs this reduction. See Figs. 10 and 11 for examples of optimal solutions constructed by the algorithm.

By Lemma 3.4, constructing an outerplanar decomposition of an area component amounts to applying the algorithm from Sect. 2. Hence, by Lemma 3.2, it suffices to find a collection of cut components so that the remainder is a suitable core component and the number of internal vertices introduced by these cut components is minimized.

We need some more terminology. Let $G^{\prime}$ be a partial conformal decomposition of polygon $P$. This means that the outer face of $G^{\prime}$ is isomorphic to $P$, and that all internal faces except one are quadrilaterals. (The exceptional face is yet to be refined.) Let $F^{\prime}$ denote this face. We call a vertex of $F^{\prime}$ relatively convex if it is either a corner of $P$, or it has degree at least three in $G^{\prime}$. A relative segment of $F^{\prime}$ is an interval of $F^{\prime}$ between two successive relative corners. By Lemma 3.4, we can 


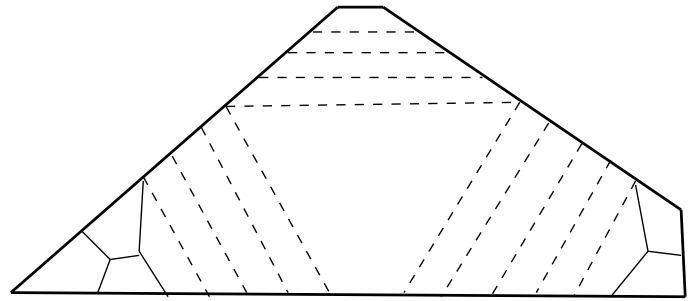

Figure 10: An optimal decomposition with one cut component of the type in Fig. 8(c) and one of the type in Fig. 8(d). The dashed lines are the chords of the (outerplanar) core component. In this example, the core component is decomposed according to a dual subdivision of $K_{1,3}$.

extend $G^{\prime}$ to a full conformal decomposition of $P$ by decomposing $F^{\prime}$ and thereby treating relatively convex vertices as corners and every other vertex of $F^{\prime}$ as a vertex having internal angle $\pi$.

Note that all cut components except those in Fig. 8(a) and (b) introduce trivial relative segments for the core component. The idea is to construct a collection of cut components such that the induced trivial relative segments allow a path decomposition or a $K_{1,3}$-decomposition.

Consequently, the algorithm consists of two stages: The first stage tries to find such a collection so that the core component admits a $K_{1,3^{-}}$ decomposition; in the second stage, the core component shall instead be a path decomposition. If succeeding, each stage determines a solution with a minimum number of vertices. By Lemmas 2.6, 2.8 , and $3.5(3)$, at least one stage will succeed, and if both stages succeed, the better result is the overall optimum.

Remember the definition of $r(\cdot)$ and $\ell(\cdot)$ for Lemma 2.9. For a real number $x \in[0, n]$, we next introduce the values $\ell_{j}(x)$ and $r_{j}(x)$ for $j=0,1,2$. This is a generalization of the values $\ell(x)$ and $r(x)$ introduced in Sect. 2. Roughly speaking, $\ell_{j}(x)\left(r_{j}(x)\right.$, resp.) denotes the clockwise (counterclockwise) distance from $x$ to the next possibility to create a trivial relative segment spending $j$ additional internal vertices. In particular, we have $\ell_{0}(x)=\ell(x)$ and $r_{0}(x)=r(x)$.

More formally, $\ell_{1}(x)$ is the first value $i$ in the sequence $(\lfloor x\rfloor,\lfloor x\rfloor-1,\lfloor x\rfloor-2, \ldots)$ such that the edges no. $i, \ldots, i-3$ may belong to a cut com-

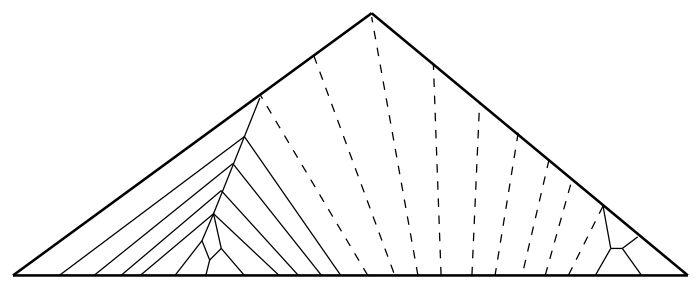

Figure 11: An optimal decomposition with one cut component of the type in Fig. 8(d) and one of the type in Fig. 9. By Lemma 3.5(6), the core component admits a path decomposition.

ponent of type (c) in Fig. 8. In other words, the vertices between the edges $i$ and $i-1$ and between the edges $i-2$ and $i-3$ are corners of $P$. Analogously, $\ell_{2}(x)$ is the first value $i$ in this sequence such that the edges no. $i, \ldots, i-3$ may belong to a cut component of type (d), or edges no. $i, \ldots, i-4$ by a component of type (e). This means that either the vertex between the edges $i-1$ and $i-2$ is a corner or the vertices between the edges $i$ and $i-1$ and the edges $i-3$ and $i-4$ are corners. The definitions of $r_{1}(x)$ and $r_{2}(x)$ are mirror-symmetric. Clearly, we can compute all these values in linear time during one single pass around $P$.

Next we explain the first stage of the algorithm in detail, that is, finding a minimum conformal decomposition such that the core component admits a $K_{1,3}$-solution. See Fig. 10 for an example where this stage succeeds and yields the overall optimum. Like in Sect. 2, we try to find optimal $i^{\prime}$ and $i^{\prime \prime}$ for each trivial segment $i$. However, here we do the same also for each possible trivial relative segment that may result from cutting off an area component of type (c),(d) or (e) in Fig. 8. For each such trivial segment or trivial relative segment $e$, we apply 9 variants on the procedure in Lemma 2.9: one for each combination $j_{1}, j_{2} \in\{0,1,2\}$. For $j_{1}=j_{2}=0$, this is exactly the algorithm in Lemma 2.9. The other variants differ (up to minor details) from this one in that $\ell_{0}$ and $r_{0}$ are replaced by $\ell_{j_{1}}$ and $r_{j_{1}}$ for $i^{\prime}$, and by $\ell_{j_{2}}$ and $r_{j_{2}}$ for $i^{\prime \prime}$. The best result of all these variants is the optimum for $e$, and the best 
result for all $e$ is the overall optimum.

It remains to explain the second stage of the algorithm in detail, that is, finding a minimum conformal decomposition such that the core component admits a path solution. See Fig. 11. Because of Lemma 3.5(7), all possibilities to apply type (a) or (b) in Fig. 8 may be tested separately. On the other hand, all possible reductions that need only components of types (c), (d) or (e) in Fig. 8 may be examined analogously to the first stage of the algorithm.

Lemma 3.5(5) and (7) say that we need at most one cut component of the type in Fig. 9, and that there is at most one further cut component, which is of type (c), (d), or (e) in Fig. 9. In other words, we may assume that one leaf of the decomposition according to Lemma 2.6 is either an original trivial segment or is created by type (c), (d) or (e) in Fig. 8. Let $i$ be the index of this (relatively) trivial segment. Then it is easy to see that, in an optimal solution, a component of Fig. 9 can only be applied to a corner incident to the segment to which the index $i+n / 2$ belongs. Therefore, at most two possibilities of this type must be examined after fixing the first trivial segment.

This concludes the sketch of the algorithm.

\section{Conformal Mesh Refinements}

As an application for the decomposition of simple polygons, we consider in this section the problem of constructing a minimum conformal refinement of a mesh in the three-dimensional space. This problem has resulted from a cooperation with an engineering company that sells CAD packages.

Throughout this section, we identify a mesh with the undirected graph $G=(V, E)$ that is induced by all corners of these polygons. In other words, each polygon is a "hole" of $G$, and since a side of a polygon may consist of more than one edge of $G$ (e.g., in Figs. 2 and 12), these polygons of $G$ are not strictly convex, but still convex.

Note that there may be holes of $G$ that do not belong to the approximation of the surface, namely holes of the surface itself. (These holes may not be convex.) Note further that the graph $G$ of a mesh need not be planar; for example, a mesh approximating the surface of a torus has genus one. Even more, a mesh may contain folding edges, that is, edges incident to more than two polygons (Fig. 12). We call a mesh homogeneous if it does not contain folding edges.

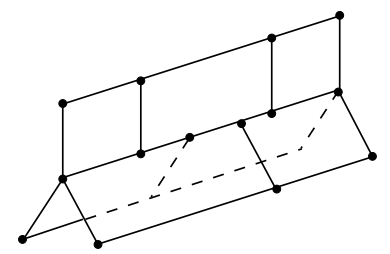

Figure 12: a small mesh with three homogeneous components and five folding edges.

Most work on conformal refinements in the literature is based on the template model, because this is easier to approach. In that model, the possibilities of decomposing a single polygon are restricted to a few classes of templates. For example, the most important template for quadrilateral polygons is an $(m \times n)$-grid, where $m$ and $n$ are variable.

Ref. [11] introduces an integer programming approach, which relies on very strong template restrictions. In [7], we reported on a cooperation with an engineering company, which sells CAD packages. In this cooperation, we developed and implemented an algorithm for mesh quadrangulation, which is based on a more flexible template model and applies network flow techniques. This algorithm performs very well in practice, but it is a pure heuristic.

In this paper now, we attack the problem from a theoretical point of view and introduce a new approach that does not rely on template restrictions.

Unfortunately, the minimum conformal mesh refinement problem is highly intractable:

Theorem 4.1 The minimum conformal mesh refinement problem is strongly $\mathcal{N} \mathcal{P}$-hard even for homogeneous meshes.

Proof: by a reduction from Exact Cover by 3Sets [3]; all details are omitted.

However, for the asymptotic complexity of the numerical analysis it is not necessary to find the exact minimum of quadrilaterals, but to achieve a constant approximation ratio. 
The following lemma shows that there is an algorithm that runs in linear time and achieves a small constant approximation ratio.

Theorem 4.2 There is a linear-time algorithm that constructs a conformal refinement of a mesh such that the number of quadrilaterals is at most four times the optimum.

Proof (sketch): Let $G=(V, E)$ denote the mesh. We subdivide each edge in $E$ exactly once. Obviously, each polygon is even afterwards, and we may apply the algorithm from Sect. 3 separately to each polygon. This already completes the description of the algorithm.

To prove the approximation ratio, we need some further terminology. For a convex polygon $P$ with an even number of vertices, $\min (P)$ denotes the minimum number of quadrilaterals required by any conformal decomposition of $P$.

For an arbitrary convex polygon $P$ with edge set $E_{P}$, a mapping $X: E_{P} \rightarrow \mathbb{N}_{0}$ is called feasible if $\sum_{e \in E_{P}} X(e)$ has the same parity as $E_{P}$. In particular, if $\left|E_{P}\right|$ is even, $X \equiv 0$ is possible, too. The polygon $P_{X}$ is constructed from $P$ by subdividing each edge $e \in E_{P}$ exactly $X(e)$ times. Hence, $X$ feasible means that $P_{X}$ admits a conformal decomposition. Moreover, $\operatorname{Min}(P)$ denotes the minimum number of quadrilaterals in any conformal decomposition of any polygon $P_{X}$,

$$
\begin{aligned}
\operatorname{Min}(P):=\min & \{q(G) \mid G \text { conformal decomposition } \\
& \text { of } \left.P_{X}, X: E_{P} \rightarrow \mathbb{N}_{0} \text { feasible }\right\} .
\end{aligned}
$$

Let $P$ be a polygon of $G$, let $X: E_{P} \rightarrow \mathbb{N}_{0}$ be uniformly equal to $1, X \equiv 1$. The heart of the proof (all details omitted; see Appendix D) is to show that $\min \left(P_{X}\right) \leq 2 \cdot \operatorname{Min}(P)+\left|E_{P}\right|-2$ for this special class of weightings $X$. Because of this fact, it suffices to prove $\left|E_{P}\right| \leq 2 \cdot \operatorname{Min}(P)+2$. However, this follows from the proof of Lemma 3.2: Let $Y: E_{P} \rightarrow \mathbb{N}_{0}$ such that $\operatorname{Min}(P)=\min \left(P_{Y}\right)$, and let $i(Y)$ denote the number of internal vertices of an optimal conformal decomposition of $P_{Y}$. Then we have $\min \left(P_{Y}\right)=i(Y)+\left|E_{P}\right| / 2-1$, and the claim follows.

\section{References}

[1] M. Bern and D. Eppstein. Mesh generation and optimal triangulation. In D.-Z. Du and F. Hwang, editors, Computing in Euclidean Geometry, pages 23-90. World Scientific, 1992.

[2] H. Everett, W. Lenhart, M. Overmars, T. Shermer, and J. Urrutia. Strictly convex quadrilateralizations of polygons. In Proceedings Fourth Canadian Conference on Computational Geometry, pages 77-83, 1992.

[3] M. R. Garey and D. S. Johnson. Computers and Intractability: $A$ Guide to the Theory of $\mathrm{NP}_{-}$ Completeness. W H Freeman \& Co Ltd, 1979.

[4] K. Ho-Le. Finite element mesh generation methods: a review and classification. Computer-Aided Design, 20:27-38, 1988.

[5] B. Joe. Quadrilateral mesh generation in polygonal regions. Computer-Aided Design, 27:209-222, 1995.

[6] A. Lubiw. Decomposing polygonal regions into convex quadrilaterals. In Proceedings of the ACM Symposium on Computational Geometry, pages 97-106, 1985.

[7] R. H. Möhring, M. Müller-Hannemann, and K. Weihe. Using network flows for surface modelling. In Proceedings of the 6th Annual ACM-SIAM Symposium on Discrete Algorithms, SODA'95, pages 350-359, 1995.

[8] T. Nishizeki and N. Chiba. Planar Graphs: Theory and Algorithms, volume 32 of Annals of Discrete Mathematics. North-Holland, 1988.

[9] J. R. Sack. An $\mathcal{O}(n \log n)$ algorithm for decomposing simple rectilinear polygons into convex quadrilaterals. In Proceedings 20th Conference on Communications, Control, and Computing, pages 64-74, 1982.

[10] J. R. Sack and G. Toussaint. A linear-time algorithm for decomposing rectilinear star-shaped polygons into convex quadrilaterals. In Proceedings 19th Conference on Communications, Control, and Computing, pages 21-30, 1981.

[11] T. Tam and C. Armstrong. Finite element mesh control by integer programming. Int. J. Numer. Methods Eng., 1993.

[12] G. Toussaint. Quadrangulations of planar sets. In Proceedings 4th International Workshop on Algorithms and Datastructures, WADS'95, Kingston, Canada, August 16-18, 1995, pages 218-227, 1995. 


\section{Appendix}

\section{A Proof of Lemma 3.4}

Remark A.1 For convenience, the proof is formulated only for the case of polygons in the plane; the extension to polygons in other smooth surfaces is straightforward. The proof disregards further interesting aspects such as internal angles of embedded quadrilaterals. However, the decompositions constructed in Sects. 2 and 3 are of a very restricted shape, and further criteria may be fulfilled to some extent, too.

Proof (sketch): The idea of the proof is to use induction on the number of internal faces $|F|$ of $G$. For technical reasons, however, we use a slightly different induction hypothesis. Let us relax the degree constraints of Item 3 in the following way:

3' Every internal vertex of $G$ has degree 3 .

4' If $F$ shares two or more vertices with another face $F^{\prime}$, these vertices form a common interval of both $F$ and $F^{\prime}$. 2.

Hence we allow non-corners of $P$ to have degree

Claim A.2 Let $P$ and $G$ and the Items 1,2, and 5 as in Lemma 3.4, but replace Item 3 by 3' and Item 4 by 4'.

Then there is a planar embedding of $G$ such that the outer face is embedded like $P$ and all internal faces are strictly convex except those which are incident to non-corners of degree 2. Nonetheless, the latter faces are still convex.

Clearly, it suffices to prove this claim, as Item 3 implies that all faces of $G$ can be embedded strictly convex.

The case $|F|=1$ is trivial. Hence, assume $|F|>$ 1.

The conditions in Lemma 3.4 imply that there is a path $P$ which connects two vertices $v$ and $w$ on the boundary of $P$ such that all edges of $p$ are internal edges. In particular, we assume that the vertices $v, w$ and the path $p$ are chosen such that $p$ is shortest subject to this condition.

As $v$ and $w$ belong to different segments of $P$ and $P$ is convex, we can embed $p$ on a straight line between $v$ and $w$ (such that the vertices of $p$ are equally spaced, say). Regard $p$ as oriented from $v$ to $w$.

Now consider the polygons $P_{\ell}=p \cup I_{P}(w, v)$, $P_{r}=p \cup I_{P}(v, w)\left(I_{P}(\cdot, \cdot)\right.$ is the counterclockwise interval with respect to $P$ ), and the subgraphs $G_{\ell}$ and $G_{r}$ which are the restrictions of $P$ and $G$ to the left hand and right hand side of $p$, respectively.

We want to apply the induction hypothesis to the convex polygon $P_{\ell}$ and $G_{\ell}$, and to $P_{r}$ and $G_{r}$. However, to this end we have to modify $p$ to ensure all preconditions. Clearly, $G_{\ell}$ and $G_{r}$ fulfill Items 1 and 2 of the preconditions, and each has fewer internal faces than $G$. It is also easy to see that the property stated in Item 5 carries over to them.

At this point it becomes clear why we used the relaxed version of Item 3: We cannot be sure that each internal vertex of $p$ has degree 3 or more in $G_{\ell}$ and $G_{r}$.

Suppose Item 4' is not valid anymore for $P_{\ell}$ or $P_{r}$, say for $P_{\ell}$. Then there is a face $f$ with two non-adjacent vertices $v_{1}, v_{2}$ on $p$. Denote by $v_{3}, v_{4}$ the two other vertices of this face, and let $v_{3}$ be the one which is enclosed by the cycle formed by the edges $\left(v_{4}, v_{1}\right),\left(v_{4}, v_{2}\right)$ and the interval between $v_{1}$ and $v_{2}$ on $p$ in the embedding of $G$. The distance of $v_{1}$ and $v_{2}$ on $p$ must be equal to 2 , as otherwise $p$ would not be a shortest path between $v$ and $w$. Let $v_{5}$ be the vertex between $v_{1}$ and $v_{2}$ on $p$. Then remove from $p$ the edges $\left(v_{1}, v_{5}\right),\left(v_{5}, v_{2}\right)$ and replace them by $\left(v_{1}, v_{3}\right),\left(v_{3}, v_{2}\right)$ to yield a new path $p^{\prime}$ between $v$ and $w$. Assume that we have chosen $p^{\prime}$ instead of $p$ in the beginning, and iterate this modification step if necessary. Observe that Item 5 now guarantees that the change from $p$ to $p^{\prime}$ cannot cause a (new) violation of Item 4' in the other subgraph. (In fact, this is the place where we need Item 5.)

Hence, we finally find $P_{\ell}$ and $P_{r}$ to which the in- 
duction hypothesis applies. Thus, both polygons admit the desired strictly convex embeddings with the exception of only those faces incident internal vertices of $p$ which have degree 2 in $G_{\ell}$ or $G_{r}$.

But now we use the fact that for any strictly convex polygon there is an $\epsilon>0$ such that we can shift any of its vertices from its current position into an arbitrary direction within some disk of ra$\operatorname{dius} \epsilon$, and the "shifted" polygon remains strictly convex. Hence, if necessary, we can readjust internal vertices of $p$ (and keep all other vertices stationary) such that all faces of $G$ are simultaneously strictly convex.

\section{B Proof of Lemma 3.5}

In this section, we give a detailed proof of Lemma 3.5. As the proof is quite long and involved and includes several extensive case distinctions, the proof is divided into three parts (Sects. B.1-B.3).

In Sect. B.1, we consider internal quadrilaterals, that is, quadrilaterals that share no edge with the boundary of the polygon (though possibly vertices). Roughly speaking, we prove that every even polygon admits an optimal conformal decomposition such that an internal quadrilateral appears only in a configuration as shown in Fig. 13 (except for the polygon $(2,1,1)$, to be precise). As a by-product (Lemma B.3), we characterize the unique optimal conformal decompositions for polygons of the type $(2 k, 1,1), k \in \mathbb{N}$.

Then, in Sect. B.2, we show that we may also restrict our attention to optimal conformal decompositions where internal vertices appear only in very specific situations. Based on this characterization, we prove that there is an optimal conformal decomposition with an area decomposition such that all but at most one area components belong to one of the types depicted in Figs. 8 and 9, the cut components.

Finally, in Sect. B.3, we prove that there is an optimal conformal decomposition such that an area decomposition contains only a few of these cut components. From this we conclude correctness of Lemma 3.5 and are done.

Throughout Sect. B, we need some further terminology. For two polygons $P=\left(s_{1}, \ldots, s_{k}\right)$ and
$Q=\left(t_{1}, \ldots, t_{\ell}\right)$ with $k>\ell$, we write $P \prec Q$ if there are $i_{0}=0<i_{1}<\cdots<i_{\ell}=k$ such that

$$
t_{j}=\sum_{i=i_{j-1}+1}^{i_{j}} s_{i}
$$

for $j=1, \ldots, \ell$. In other words, $Q$ may be constructed from $P$ by "stretching" some strictly convex internal angles to $\pi$. Obviously, “ $\prec$ " is a partial order. We write $P \preceq Q$ if $P \prec Q$ or $P=Q$.

\section{B.1 Internal Quadrilaterals}

Lemma B.1 is auxiliary (nonetheless insightful), Lemma B.2 shows that internal quadrilaterals appear only in configurations as depicted in Fig. 13, and Lemma B.3 characterizes the optimal conformal decompositions of polygons $(2 k, 1,1)$.

Lemma B.1 No two internal quadrilaterals are adjacent in an optimal conformal decomposition if at least one of them is incident to an internal vertex.

Proof: Let $Z$ be be an inclusion-maximal connected component of internal quadrilaterals (connectivity with respect to the reduced dual graph $G^{*}$, see Sect. 2). Suppose for a contradiction that $Z$ is incident to an internal vertex and consists of more than one quadrilateral.

Let $C$ be the cycle separating $Z$ from the rest of the plane, and let $e_{1}=\left(v_{0}, v_{1}\right), e_{2}=$ $\left(v_{1}, v_{2}\right), \ldots, e_{k}=\left(v_{k-1}, v_{0}\right)$ be the edges of $C$ (in this counterclockwise cyclic order). Moreover, let $V_{1}, \ldots, V_{k}$ be the quadrilaterals incident to $e_{1}, \ldots, e_{k}$ outside $Z$. (Observe that these "outer" quadrilaterals are not necessarily pairwise different.)

We show in the following that we can always modify the given conformal decomposition such that $L(C)$ shrinks by at least two unless $L(C)=4$. This suffices for an induction on $L(C)$.

Case I: No two quadrilaterals $V_{i}$ are identical.

Then for all $v_{i}, i=0, \ldots, k-1$, there is an edge leaving $Z$ at $v_{i}$. Thus $Z$ itself may be treated like a polygon that is everywhere strictly convex (and thus admits a perfect conformal decomposition).

Case I(a): $V_{i}$ is adjacent to $V_{i+1}$ for all $i=$ $0, \ldots, k-1$ (with indices taken modulo $k$ ). 
For $i=0, \ldots, k-1$ let $e_{i}^{\prime}$ be the edge opposite to $e_{i}$ on $V_{i}$. Then $e_{1}^{\prime}-e_{2}^{\prime}-\cdots-e_{k}^{\prime}-e_{1}^{\prime}$ is the original polygon $P$ itself. Hence, there must be an index $\ell$, such that the common vertex of $e_{\ell}^{\prime}$ and $e_{\ell+1}^{\prime}$ is a corner. Now identify the vertices $v_{\ell-1}$ and $v_{\ell+1}$, and delete vertex $v_{\ell}$ and all its incident edges. This lets $L(C)$ shrink by two, but $Z$ remains perfect.

Case I(b): At least two consecutive quadrilaterals are not adjacent.

Then there is $i \in\{0, \ldots, k-1\}$ such that $V_{i}$ and $V_{i+1}$ are not adjacent and $v_{i-1}$ is an internal vertex. To see this, distinguish two cases: If no two consecutive quadrilaterals are adjacent, $v_{i-1}$ may be an arbitrary internal vertex (whose existence is assumed in Lemma B.1). Otherwise, there is $v_{i-1}$ such that $V_{i-1}$ and $V_{i}$ are adjacent and $V_{i}$ and $V_{i+1}$ are not. Clearly, when $V_{i-1}$ and $V_{i}$ are adjacent, $v_{i-1}$ is internal.

Now we know that there is $i \in\{0, \ldots, k-1\}$ such that $V_{i}$ and $V_{i+1}$ are not adjacent and $v_{i-1}$ is an internal vertex. Then the vertices $v_{i-1}$ and $v_{i+1}$ can be identified with each other. As a result, $L(C)$ shrinks by two, but $Z$ still allows a perfect conformal decomposition.

Case II: There is an index $i$ with $V_{i}=V_{i+1}$.

Case II(a): $V_{i} \neq V_{i-1}$ and $V_{i+1} \neq V_{i+2}$.

The quadrilateral $V_{i}$ is incident to the edges $e_{i}$ and $e_{i+1}$. Denote by $e_{i}^{\prime}$ and $e_{i+1}^{\prime}$ the opposite sides of $e_{i}$ and $e_{i+1}$ in $V_{i}$. Since $Z$ is inclusionmaximal, $V_{i}$ is not an internal quadrilateral, so $e_{i}^{\prime}$ or $e_{i+1}^{\prime}$ (say $e_{i+1}^{\prime}$ ) belongs to the boundary of $P$. The vertex $v_{i}$ is strictly convex relative to the union of $Z$ and $V_{i}$. Thus, we may remove $e_{i}$ and $e_{i+1}$ and replace them by an edge $e=\left(v_{i-1}, v_{i+2}\right)$, thereby creating a new quadrilateral $V$ with edges $e, e_{i-1}, e_{i}^{\prime}, e_{i+1}^{\prime}$. The modified $C$ has been shrunk by two edges, and outside $C$ nothing changes.

Case II(b): $V_{i}=V_{i+1}=V_{i+2}$.

In this case, $e=\left(v_{i-1}, v_{i+2}\right)$ is a boundary edge of $P$, and $v_{i-1}$ and $v_{i+2}$ are both strictly convex relative to the union of $Z$ and $V_{i}$. Delete the vertices $v_{i}$ and $v_{i+1}$ with all their incident edges, and insert a new edge $e^{\prime}=\left(v_{i-2}, v_{i+3}\right)$. This edge $e^{\prime}$ is feasible, as neither $v_{i-2}$ nor $v_{i+3}$ can belong to the same segment as $e$. By that, $V_{i}$ is re-

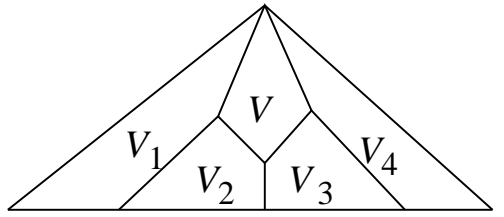

Figure 13: Lemma B.2 means that an internal quadrilateral $V$ occurs only in a configuration like this, where the four horizontal edges below are consecutive edges of the same segment of the polygon.

placed by the quadrilateral consisting of the four edges $e, e_{i-1}, e^{\prime}, e_{i+4}$. This time, $L(C)$ shrinks by 4 units. (Note that $L(C) \geq 8$ before this modification.)

Lemma B.2 Let $P \neq(2,1,1)$ be a convex polygon, and let $G$ be an optimal conformal decomposition of $P$ with a minimum number of internal quadrilaterals. Assume that $G$ contains an internal quadrilateral $V$ that is incident to at least one internal vertex, and let $V$ be adjacent to the quadrilaterals $V_{1}, V_{2}, V_{3}, V_{4}$ in cyclic order. Then (for an appropriate choice of $V_{1}$ ) $V_{i}$ and $V_{i+1}$ are adjacent for $i=1,2,3$, and all four quadrilaterals are incident to consecutive edges of the same segment of $P$ (see Fig. 13).

Proof: By Lemma B.1 we may assume that no $V_{i}$ is an internal quadrilateral. Let $e_{i}=\left(w_{i}, w_{i+1}\right)$ be the edge incident to both $V$ and $V_{i}$ for $i=1, \ldots, 4$ (with $w_{5}=w_{1}$ ). Furthermore, denote by $e_{i}^{\prime}$ the opposite edge of $e_{i}$ in $V_{i}$.

Case I: degree $\left(w_{i}\right)=3$ for $i=1, \ldots, 4$.

As no $V_{i}$ is an internal quadrilateral, all edges $e_{i}^{\prime}$ lie on the boundary of $P$. By assumption, $P \neq(2,1,1)$, thus $P=(1,1,1,1)$, and this case contradicts optimality.

Case II: At least three vertices, say $w_{1}, w_{2}, w_{3}$, have degree $\geq 4$.

Then $w_{4}$ can be assumed to be the internal vertex of $V$, and therefore be identified with $w_{2}$. This gives a feasible conformal decomposition with fewer internal vertices.

Case III: Exactly two opposite vertices, say $w_{1}$ and $w_{3}$, have degree $\geq 4$. 
Then the identification of $w_{2}$ and $w_{4}$ gives an improved feasible conformal decomposition.

Case IV: Two adjacent vertices, say $w_{1}$ and $w_{2}$, have degree $\geq 4$, and $w_{3}, w_{4}$ have degree $=3$.

Case IV(a): $\operatorname{degree}\left(w_{2}\right)>4$. (The case degree $\left(w_{1}\right)>4$ is mirror-symmetric.)

The solution can always be improved, see Fig.14. Drawn is the "worst case", i.e. the case with the smallest number of strictly convex vertices. Hence, the following solution works for all other cases, too.

First identify $w_{2}$ and $w_{4}$, then delete $w_{3}$ and all its incident edges. Delete furthermore the first two edges of the cyclic adjacency list of $w_{2}$ between $\left(w_{2}, w_{3}\right)$ and $\left(w_{2}, w_{1}\right)$. Introduce a new edge $\left(w_{3}^{\prime}, z\right)$, and complete the construction in the obvious way.

Case IV(b): degree $\left(w_{1}\right)=\operatorname{degree}\left(w_{2}\right)=4$.

First suppose, that there is a quadrilateral $V_{5}$ neighbored to $V_{1}$ and to $V_{2}$, and let $z$ the vertex incident to both $V_{1}$ and $V_{5}$. If $z$ is strictly convex relative to the subpolygon $Q_{1}$ formed by the quadrilaterals $V, V_{1}, \ldots, V_{5}$ then we can find a solution for $Q_{1}$ which needs only 2 internal vertices. If $z$ is not strictly convex, but $z$ is an internal vertex, then there is a third quadrilateral $V_{6}$ adjacent to $z$. The subpolygon $Q_{2}=Q_{1} \cup V_{6}$ currently uses 4 internal vertices. However, the two possibilities with fewest segments $Q_{2}=(5,2,1)$ or $Q_{2}=(4,3,1)$ can be solved with less internal vertices. Now consider the case, that $z$ is on the boundary of $P$ and not strictly convex. If $w_{1}$ is also on the boundary, the possibility $Q_{1}=(4,2,2)$ has an optimal solution without internal quadrilateral, and otherwise $Q_{1} \prec(4,2,2)$ needs less internal vertices than the current solution. If $w_{1}$ is an internal vertex, we have the same subcases as with $Q_{2}$.

It remains the case, where $V_{5}$ does not exist. In other words, $w_{2}$ lies on the boundary of $P$. By symmetry, we may also assume, that $w_{1}$ lies on the boundary. But then, we can find an alternative solution for the quadrilaterals $V, V_{1}, \ldots, V_{4}$ which uses the same number of internal vertices, but no internal quadrilateral.
Case V: $\operatorname{degree}\left(w_{2}\right)=\operatorname{degree}\left(w_{3}\right)=$ $\operatorname{degree}\left(w_{4}\right)=3$, degree $\left(w_{1}\right) \geq 4$.

Case V(a): The common vertex of $e_{1}^{\prime}$ and $e_{2}^{\prime}$ is strictly convex relative to $Q_{3}=V \cup V_{1} \cup V_{2} \cup V_{3} \cup V_{4}$. Then $Q_{3} \preceq(3,1,1,1)$, and so needs at most two internal vertices.

Case V(b): The common vertex of $e_{2}^{\prime}$ and $e_{3}^{\prime}$ is strictly convex relative to $Q_{3}$.

In this case, $Q_{3} \preceq(2,2,1,1)$, and so needs at most one internal vertex.

Case V(c): All 4 quadrilaterals $V_{1}, \ldots, V_{4}$ are incident to the same segment of $P$.

This final case was stated in the lemma.

Lemma B.3 The optimal conformal decomposition of the polygon $(2 k, 1,1)$ is unique for all $k \geq 1$. The polygon $(2,1,1)$ needs four internal vertices, and for $k \geq 2$, the polygon $(2 k, 1,1)$ needs $k+1$ internal vertices.

Proof: The proof in done by induction on $k$. Denote by $a, b, c$ the strictly convex vertices of the polygon $(2 k, 1,1)$, and let $v_{1}, v_{2}, \ldots, v_{2 k-1}$ be the remaining vertices on the base segment between $a$ and $b$.

In any solution, each edge of the base segment is incident to one quadrilateral, and all of them have to be pairwise different. This gives an immediate lower bound of $2 k$ quadrilaterals for any conformal decomposition. For $k \geq 2$, the given solution needs exactly $2 k+1$ quadrilaterals. Hence, a solution cannot be optimal if it uses 2 quadrilaterals not incident to the base segment in this case.

Case $k \leq 2$ :

Easy to see.

Case $k>2$ :

Let $G$ be an optimal conformal decomposition. If degree $(a)=$ degree $(b)=2$ and degree $(c)=$ 3 , then there is a vertex $v$ incident to $c$ and $v_{1}, v_{2 k-1}$. This means that the induced subpolygon $\left(v, v_{1}, \ldots, v_{2 k-1}, v\right)$ has the shape of a $(2 k-$ $2,1,1)$, and we are done by induction.

Suppose degree $(a)>2$. This implies the existence of a quadrilateral $V_{1}=\left(a, v_{a}, v_{c}, c\right)$ not incident to the base segment. The internal vertex $v_{a}$ must have degree $\geq 3$, and therefore be incident to an edge $\left(v_{a}, v_{d}\right)$ (with $a \neq v_{d} \neq v_{c}$ ). If $v_{d}$ can be chosen as an internal vertex, then we may also 

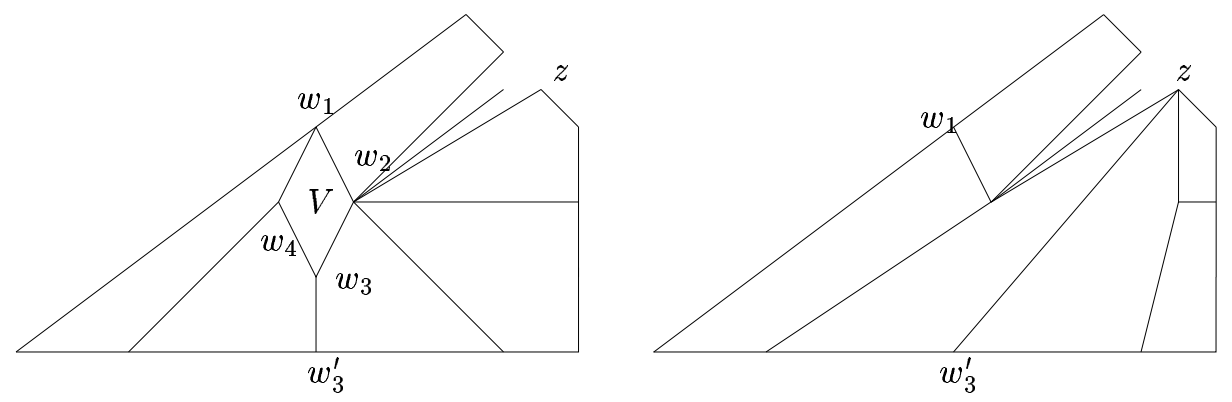

Figure 14: Case IV(a) in Lemma B.2.

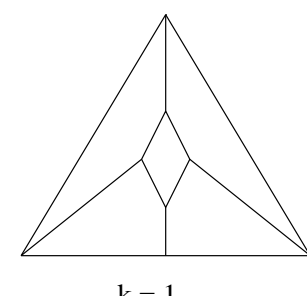

$\mathrm{k}=1$

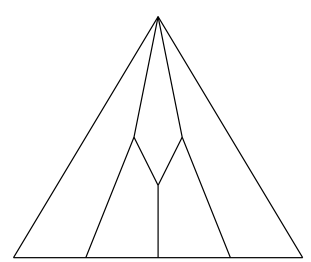

$\mathrm{k}=2$

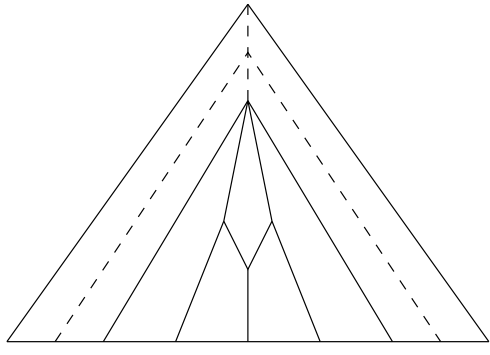

$\mathrm{k}>2$

Figure 15: Illustration of optimal conformal decompositions of $(2 k, 1,1)$

assume that $v_{d}, v_{a}$ and $v_{c}$ belong to a further internal quadrilateral $V_{2}$. If $v_{d}$ belongs to the boundary of $P$, then this implies a triangle-shaped subpolygon $\left(2 k^{\prime}, 1,1\right)$ with $a, v_{a}, v_{d}$ as strictly convex vertices. By induction hypothesis (note that $v_{d} \neq b$ ), this subpolygon needs at least one internal quadrilateral. Thus, the case degree $(a)>2$ is always suboptimal, and by symmetry this holds also for degree $(b)>2$. But if degree $(a)=\operatorname{degree}(b)=2$, we have necessarily $\operatorname{degree}(c) \geq 3$.

It remains to show that degree $(c)>3$ is suboptimal. Any solution which includes an edge $\left(c, v_{i}\right)$ cannot be optimal, as such an edge either induces two odd polygons (which is infeasible) or induces two triangle shaped polygons for which the induction hypothesis applies and so is suboptimal. If degree $(c)>4$, the solution has two internal quadrilaterals which are adjacent. By Lemma B.1, this is not optimal. Thus, the only interesting case is degree $(c)=4$. Let $(c, d)$ and $(c, e)$ be the two edges starting at $c$ and going into the internal of $P$. Clearly both edges are incident to an internal quadrilateral $V$. Denote by $f$ the fourth vertex of $V$. Then $f$ must be an internal vertex and degree $(f)=3$, as otherwise $d$ and $e$ could be identified. In addition, there are edges $\left(v_{1}, d\right)$ and $\left(v_{2 k-1}, e\right)$, because degree $(a)=\operatorname{degree}(b)=2$. Furthermore, we cannot have degree $(d) \geq 4$ and degree $(e) \geq 4$, as otherwise $f$ and $c$ could be identified. Suppose therefore that degree $(d)=3$. But this implies the existence of edges $\left(v_{2}, f\right)$ and $\left(v_{3}, e\right)$, as no internal quadrilateral can be adjacent to $V$ in an optimal conformal decomposition. But now $v_{3}, e, v_{2 k-1}$ induce a triangle-shaped subpolygon for which the induction hypothesis can be applied. This observation finishes the induction step.

\section{B.2 Internal Components}

For the set of operations illustrated in Fig. 8 we use the following terminology.

Terminology B.4 Operation (a) in Fig. 8 is called a prolongation as it enlarges the length of some interval. Operation (b) has (possibly) a negative effect on the reduced length $(L-2 K)$ and is therefore said to be a 1-reduction. Those operations which create relatively trivial segments 


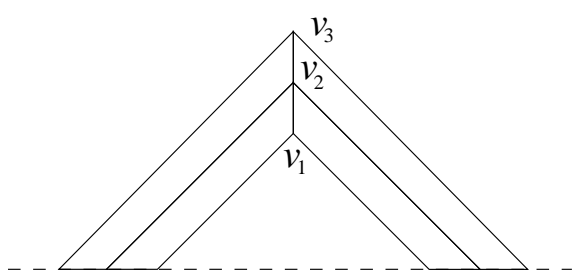

Figure 16: Lemma B.6 says that, if two incident edges $\left(v_{1}, v_{2}\right),\left(v_{2}, v_{3}\right)$ between internal vertices are not incident to the same internal quadrilateral, then they form a constellation like this.

are called cuts, operation (c) is a 1-cut, whereas operations (d) and (e) are 2-cuts. Finally, the cuts described in Fig. 9 are called $(2 k, 1,1)$-cuts. $A(2 k, 1,1)$-cut requires a segment $S$ of length $L(S) \geq 2 k$, which is henceforth called the base segment of this cut.

Observation B.5 Let $G$ be a feasible conformal decomposition of the polygon $P$. If $v$ is an internal vertex of $G$ and connected to vertices $v_{1}$ and $v_{2}$ on the same segment of $P$, then the edges $\left\{v, v_{1}\right\}$ and $\left\{v, v_{2}\right\}$ are not incident to a common face.

Fig. 13 shows that $v_{1}$ and $v_{2}$ may be on the same segment if $\left\{v, v_{1}\right\}$ and $\left\{v, v_{2}\right\}$ are not incident to a common face.

The following three lemmas, Lemma B.8, B.9, and B.6, restrict the ways internal vertices may occur in optimal conformal decompositions.

But first, we need some more terminology. For a conformal decomposition $G$ of polygon $P$ let $G^{i}$ denote the subgraph induced by all internal vertices. We call an internal vertex isolated if it is not incident to another internal vertex. Analogously, an edge of $G^{i}$ is isolated if it is not incident to any other edge of $G^{i}$. Lemma B.6 characterizes the connected components of $G^{i}$ that are not isolated vertices or edges. Afterwards, this result is used to characterize the area components of $G$.

Lemma B.6 For any polygon $P \notin\{(3,3,3,3)$, $(4,3,3),(4,2,2)\}$, there is an optimal conformal decomposition $G$ such that for any two incident edges $\left(v_{1}, v_{2}\right),\left(v_{2}, v_{3}\right)$ in $G^{i}$ one of the following holds:

- Either at least one of $\left(v_{1}, v_{2}\right)$ and $\left(v_{2}, v_{3}\right)$ is incident to an internal quadrilateral,
- or the configuration in Fig. 16 occurs: All four incident quadrilaterals are pairwise different, and the four edges opposite to $\left(v_{1}, v_{2}\right)$ and $\left(v_{2}, v_{3}\right)$ belong to the same segment of $P$.

Proof: Let $G$ be an optimal conformal decomposition of $P$. For internal components which are isolated vertices or single edges nothing is to show. We treat all larger internal components by an extensive case distinction. Throughout this proof we may assume that internal quadrilaterals of $G$ (if any) are of the form described in the statement of Lemma B.2. Thus let $v_{1}, v_{2}, v_{3}$ be internal vertices of $G$ which are connected by the edges $\left(v_{1}, v_{2}\right)$ and $\left(v_{2}, v_{3}\right)$. If one of the quadrilaterals incident to $\left(v_{1}, v_{2}\right)$ or to $\left(v_{2}, v_{3}\right)$ is an internal quadrilateral, the lemma holds. Hence, in the following we assume the converse.

Case I: degree $\left(v_{2}\right)=3$.

This means that two quadrilaterals, say $V_{1}$ and $V_{3}$, coincide. But then $V_{1}$ is an internal quadrilateral, a contradiction.

Case II: $\operatorname{degree}\left(v_{1}\right)=\operatorname{degree}\left(v_{3}\right)=3$, degree $\left(v_{2}\right) \geq 4$.

Consider the bounding cycle $C$ of all quadrilaterals which are incident to $v_{1}, v_{2}, v_{3}$.

Case II(a): degree $\left(v_{2}\right)=4$.

$C=\left(a-b-c-w^{\prime}-f-e-d-w-a\right)$, with vertices named as in Fig. 17. We may assume, that the vertices $a, b, \ldots, f$ all lie on the boundary of $P$, as otherwise there is an internal quadrilateral as stated in the lemma. Because of Observation B.5, the edges $(a, b)$ and $(d, e)$, as well as $(b, c)$ and $(e, f)$ lie on different segments of $P$. If $C \preceq(4,3,1)$, there is a better solution for $C$ with only two internal vertices. Hence, none of the vertices $a, d, c, f$ is strictly convex relative to $C$. But then $P \preceq(2,2,2,2)$ which has also an improved solution, or we have the exception $P=(4,2,2)$.

Case II(b): degree $\left(v_{2}\right)=5$.

Similar to case $2 \mathrm{a})$, take the cycle $C=(a-$ $\left.b_{1}-w^{\prime \prime}-b_{2}-c-w^{\prime}-f-e-d-w-a\right)$. See again Fig. 17. Either we have the exception $P=$ $(4,3,3)$, or some additional vertex is strictly convex relative to $C$. But then the four possibilities 

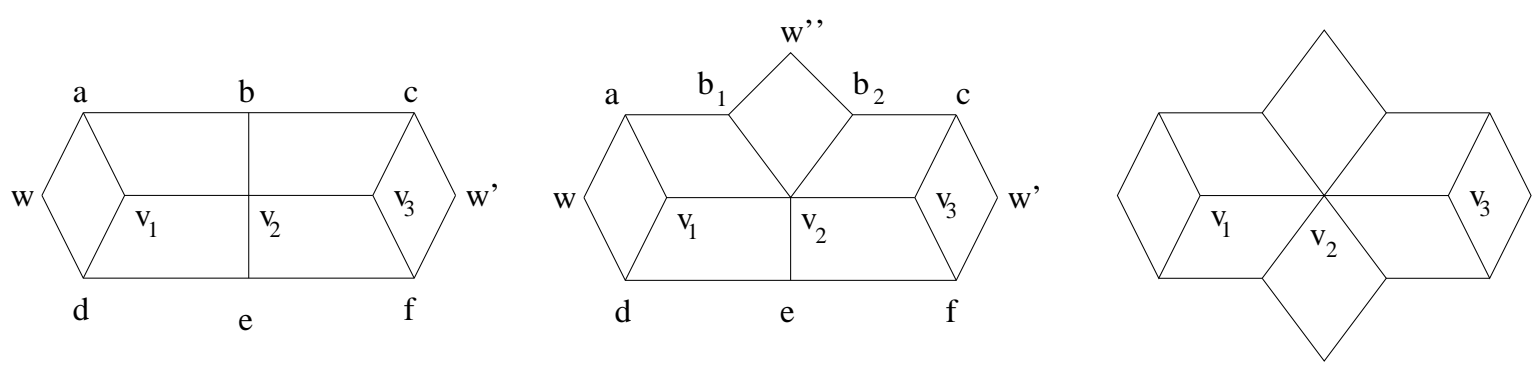

Figure 17: Characterization of internal components - Cases II(a) - II(c) in Lemma B.6
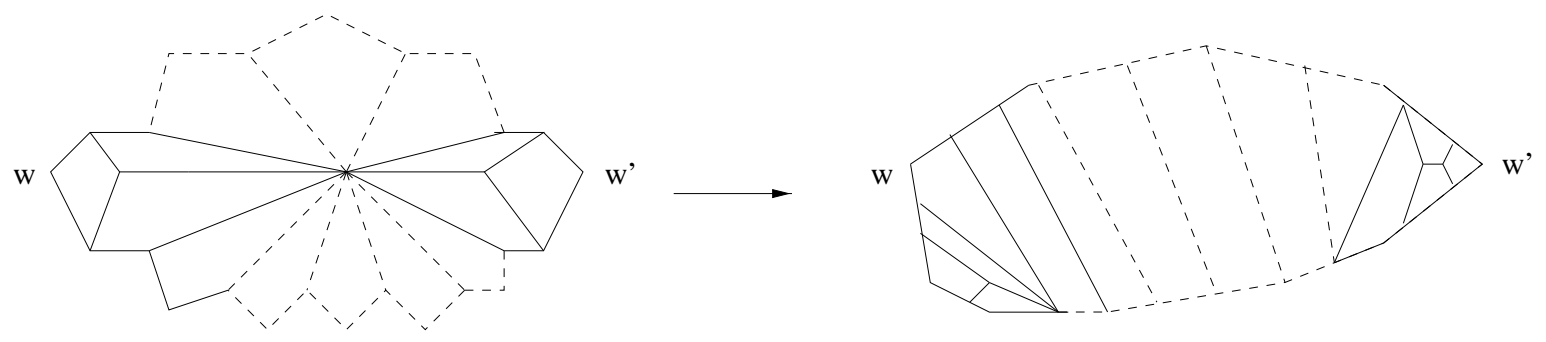

Figure 18: Characterization of internal components - Case II(d) in Lemma B.6 before and after modification

$C \preceq(3,3,2,2), C \preceq(3,3,3,1), C \preceq(4,3,2,1)$, or $C \preceq(4,3,1,2)$ all allow solutions with at most two internal vertices.

Case II(c): $\operatorname{degree}\left(v_{2}\right)=6$, and exactly two edges in the cyclic adjacency list of $v_{2}$ are between the edges $\left(v_{1}, v_{2}\right)$ and $\left(v_{3}, v_{2}\right)$.

We may have the exception $P=(3,3,3,3)$, or $C \preceq(3,3,3,2,1)$, and needs at most 2 internal vertices.

Case II(d): $\operatorname{degree}\left(v_{2}\right)=k+\ell \geq 6$, with $k \geq 3$, and exactly $k$ edges in the cyclic adjacency list of $v_{2}$ are between the edges $\left(v_{1}, v_{2}\right)$ and $\left(v_{3}, v_{2}\right)$.

Let $V_{5}\left(V_{6}\right)$ be the third quadrilateral incident to $v_{1}\left(v_{3}\right)$, and call $w\left(w^{\prime}\right)$ the vertex incident to $V_{5}\left(V_{6}\right)$ but not adjacent to $v_{1}\left(v_{3}\right)$.

The third and fifth vertex on the interval $I\left(w, w^{\prime}\right)$ on $C$ must be strictly convex relative to $C$ (they exist by the condition defining Case II(d). Hence we can apply a 1-cut based between the third and fifth vertex. In addition we apply a $2-$ cut at $w^{\prime}$. By that, $C$ can be reduced to a polygon of the form $(1,1,1,2, \ldots, 2,1,1,1,2, \ldots, 2)$, which is clearly a perfect polygon with a path solution. Moreover, there is a path solution such that no inserted edge has both endvertices on $I\left(w, w^{\prime}\right)$ or on $I\left(w^{\prime}, w\right)$. This means that all edges are feasible (they do not lie on the same segment) by Observation B.5, applied to $v_{1}$ and resp. to $v_{3}$. We have to show that we do not create a new component of internal vertices by one of these new edges. Suppose we do. Then such an edge must be incident to a vertex which has not been adjacent to $v_{1}, v_{2}, v_{3}$ before. But this would imply an internal quadrilateral in $G$ with two vertices of degree larger than 3 , in contradiction to our assumption that $G$ fulfills Lemma B.2.

Finally, the component of $v_{1}, v_{2}, v_{3}$ has been split by the new decomposition of $C$. This finishes case 2).

In all remaining cases we may assume that degree $\left(v_{3}\right) \geq 4$. Consider Fig. 19 .

The edges $\left(a_{1}, a_{2}\right)$ and $\left(b_{1}, b_{2}\right)$ lie on the boundary of $P$. To see this, observe that if $\left(a_{1}, a_{2}\right)$ does not lie on the boundary of $P$ then the quadrilateral $V$ formed by $\left(a_{1}, v_{3}, v_{2}, a_{2}\right)$ is an internal quadrilateral with $\operatorname{degree}\left(v_{2}\right) \geq 4$ and degree $\left(a_{1}\right) \geq 4$ which contradicts the situation of Lemma B.2. By symmetry, the same holds for $\left(b_{1}, b_{2}\right)$.

If $\left(a_{1}, a_{2}\right)$ and $\left(b_{1}, b_{2}\right)$ do not lie on the same segment of $P$ we may replace the edge $\left(v_{2}, v_{3}\right)$ by 


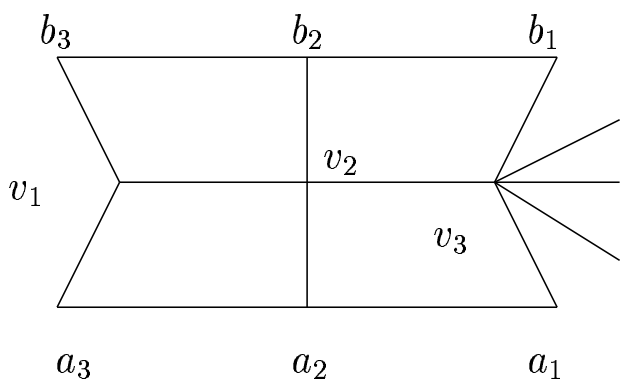

Figure 19: Characterization of internal components — Case III in Lemma B.6
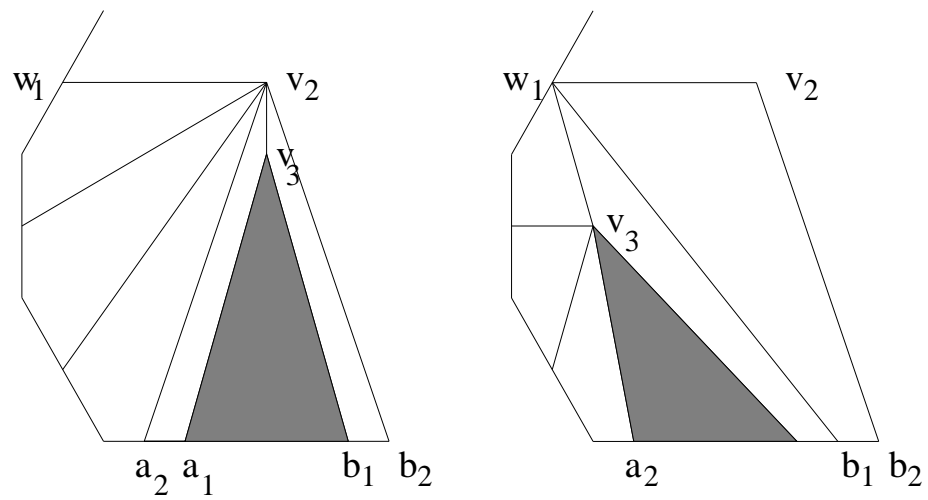

Figure 20: Characterization of internal components - Case IV in Lemma B.6: before and after the modification.

$\left(b_{1}, a_{2}\right)$. By that modification, the internal vertices $v_{2}$ and $v_{3}$ are no longer connected, and no new component is produced, as both $b_{1}$ and $a_{2}$ lie on the boundary of $P$. Hence from now on we will assume that both edges $\left(a_{1}, a_{2}\right)$ and $\left(b_{1}, b_{2}\right)$ belong to the same segment of $P$.

Case III: degree $\left(v_{2}\right)=4$ and degree $\left(v_{3}\right) \geq 4$.

If furthermore degree $\left(v_{1}\right) \geq 4$, then with the same argument as before the edges $\left(a_{2}, a_{3}\right)$ and $\left(b_{2}, b_{3}\right)$ also may be assumed to belong to the same segment of $P$. But that means that $V_{1}, V_{2}, V_{3}, V_{4}$ all are incident to the same segment of $P$, as claimed. Otherwise, degree $\left(v_{1}\right)=3$. If either $b_{3}$ or $a_{3}$ are strictly convex relative to $C$, we may improve our current solution by deleting $v_{1}$ from $G$ with all its incident edges and by insertion of the edge $\left(a_{2}, b_{3}\right)$ or $\left(b_{2}, a_{3}\right)$. If neither $b_{3}$ or $a_{3}$ are strictly convex, then both $b_{2}$ and $a_{2}$ must be strictly convex, as each polygon has at least three corners. Hence, we may delete both $v_{1}$ and $v_{2}$, and insert edges $\left(a_{3}, v_{3}\right)$ and $\left(b_{3}, v_{3}\right)$, yielding an improved conformal decomposition.

Case IV: degree $\left(v_{2}\right)>4$ and degree $\left(v_{3}\right) \geq 4$.

Suppose that $k>1$ vertices in the cyclic adjacency list of $v_{2}$ are between $v_{1}$ and $v_{3}$. Call them $w_{1}, w_{2}, \ldots, w_{k}\left(=a_{2}\right)$. As neither $V_{1}$ nor $V_{2}$ are internal quadrilaterals, $w_{1}$ is a boundary vertex of $P$.

Suppose that the vertices $w_{1}$ and $b_{1}$ do not lie on the same segment of $P$. Then the following modification is feasible, see Fig. 20. "Shift" the $(2 k, 1,1)$ formed by the interval $I\left(a_{1}, b_{1}\right)$ and the edges $\left(b_{1}, v_{3}\right)$ and $\left(v_{3}, a_{1}\right)$ by one edge in direction to $a_{2}$. Replace the edges $\left(w_{i}, v_{2}\right)$ by $\left(w_{i}, v_{3}\right)$ for $i=2, \ldots, k$, and insert edges $\left(w_{1}, v_{3}\right)$ and $\left(w_{1}, b_{1}\right)$. By that, we have again a feasible conformal decomposition, and $v_{2}$ and $v_{3}$ are no longer in the same component of internal vertices. Note that 
by the insertion of new edges, no vertices become member of the same component which have not been before.

It remains to show that we may assume that $w_{1}$ and $b_{1}$ do not belong to a common segment. First, consider the subcase that the vertex $w_{1}$ lies on the same segment of $P$ as the edge $\left(b_{1}, b_{2}\right)$, and thereby induces a $(2 k, 1,1)$ in the current solution, namely formed by the edges $v_{2}, b_{2}$ and $v_{2}, w_{1}$ and the interval $I\left(w_{1}, b_{2}\right)$ of $P$. But the current solution looks different from the unique optimal solution of such polygons we have shown in Lemma B.3. By the same argument it would also be suboptimal if $w_{1}$ lies on the same segment of $P$ as the edge $\left(b_{1}, b_{2}\right)$, and thereby induces a $(2 k, 1,1)$ in the current solution, in this case formed by $v_{2}, a_{2}$, $v_{2}, w_{1}$ and the interval $I\left(a_{2}, w_{1}\right)$ of $P$.

Corollary B.7 There is a conformal decomposition $G$ of the polygon $P$ with minimum $q(G)$ such that a connected component of $G^{i}$ which contains more than two vertices induces a subgraph in $G$ which is isomorphic to the optimal decomposition of some $(2 k, 1,1)$ in $G$, and one of the trivial segments of the $(2 k, 1,1)$ is an edge of $P$.

Proof: By Lemma B.6, there is an optimal decomposition such that for each connected component of $G^{i}$ with more than two vertices any two incident edges form a situation as depicted in Fig. 13 or 16. Denote by $G^{\prime}$ the area component of $G$ which is induced by all quadrilaterals incident to a vertex of some connected component of $G^{i}$ with more than two vertices. (If no such exists, nothing has to be proved.) By Lemma B.6, this $G^{\prime}$ contains a $(2 k, 1,1)$. Let $P_{T}$ be the largest $(2 k, 1,1)$ (by inclusion) contained in $G^{\prime}$.

The lemma claims that we may assume that one of the trivial segments of $P_{T}$ lies on $P$. To see this, suppose that both trivial segments are internal edges. Then there is a quadrilateral $Q$ incident to one of these two trivial segments but not contained in $P_{T}$. Now we have two subcases. Either the union of $P_{T}=(2 k, 1,1)$ and $Q$ forms a $(2 k+1,1,1,1)$ or it forms a $(2 k, 1,1,1,1)$ relative to $P$. It is easy to see that the latter case contradicts the optimality of the current decomposition. In the first case, however, we can modify our current decomposition by exchanging $Q$ and
$P_{T}$ within the $(2 k+1,1,1,1)$. By that, either one trivial segment of $P_{T}$ immediately becomes a boundary edge of $P$ or we can continue in the same way. If we choose always the same trivial segment in these modifications steps, then this procedure clearly stops after a finite number of steps.

Lemma B.8 There is an optimal decomposition such that degree $(v)=3$ for all isolated internal vertices, unless $P=(2,2, \ldots, 2)$ (i.e., an arbitrary number of segments, each of length 2). This decomposition has also the properties of Corollary B.7.

Proof: Suppose we have a situation where an isolated internal vertex $v$ has $\operatorname{degree}(v) \geq 4$. Let $C$ be the surrounding cycle of the set of all quadrilaterals incident to $v$. If $C \neq(2,2, \ldots, 2)$ we have $C \prec(2,2, \ldots, 2)$, because of Observation B.5. Thus, $C \preceq(1,1,2,2, \ldots, 2)$ contains two trivial segments. Let $v_{1}, v_{2}, \ldots, v_{k}$ be the vertices adjacent to $v$ in $G$ in cyclic order. Assume that $v_{1}$ is the vertex which is incident to the two trivial segments of $C$. Let $v^{\prime}$ be the vertex on $C$ between $v_{k}$ and $v_{1}$. Replace the edges $\left(v, v_{1}\right),\left(v, v_{2}\right), \ldots,\left(v, v_{k-3}\right)$ by edges $\left(v^{\prime}, v_{2}\right),\left(v^{\prime}, v_{3}\right), \ldots,\left(v^{\prime}, v_{k-3}\right)$. This modification still yields a conformal decomposition, but now we have $\operatorname{degree}(v)=3$. Observe that $v^{\prime}$ cannot be an internal vertex of $G$, as otherwise $G$ contains the internal quadrilateral $\left(v, v_{k}, v^{\prime}, v_{1}\right)$, in contradiction to Lemma B.2, Case II. This means that our modification cannot create a new violation of the claim with $v^{\prime}$ instead of $v$, and does not change the properties of other internal vertices.

Note that the exception $P=(2,2, \ldots, 2)$ does not contradict Lemma 3.5, as its (unique) optimal decomposition can be interpreted as a single 1-cut plus a perfect core polygon.

Lemma B.9 There is an optimal decomposition such that for each component of $G^{i}$ which is a single edge, say $e=\left(v_{1}, v_{2}\right)$, we have degree $\left(v_{1}\right)=$ degree $\left(v_{2}\right)=3$, unless $P=(3,3,2,2, \ldots, 2)$. This decomposition has also the properties of Corollary B.7 and Lemma B.9. 
Proof: Take an arbitrary optimal decomposition of $P$ and suppose $e=\left(v_{1}, v_{2}\right)$ is a connected component of $G^{i}$. Let $C$ be the surrounding cycle formed by all quadrilaterals incident to $v_{1}$ and $v_{2}$. We may assume that degree $\left(v_{1}\right) \geq \operatorname{degree}\left(v_{2}\right)$.

Case I: $\operatorname{degree}\left(v_{1}\right)>3$ and degree $\left(v_{2}\right)>3$.

Delete the edge $e$ and replace it by some edge $\left(w, w^{\prime}\right)$ which makes all faces to quadrilaterals. By Observation B.5, this modification is feasible, and $v_{1}$ and $v_{2}$ are now isolated.

Case II: degree $\left(v_{1}\right)>3$ and degree $\left(v_{2}\right)=3$, and some vertex $w$ incident to $v_{2}$ is strictly convex relative to $C$.

Then the current solution cannot be optimal, as we may replace $v_{2}$ and all its incident edges by a single edge.

Case III: degree $\left(v_{1}\right)>3$ and degree $\left(v_{2}\right)=3$, and some vertex $w$ incident to $v_{1}$ is strictly convex relative to $C$.

In this case, replace the edge $\left(w, v_{1}\right)$ by a new one, say $\left(w^{\prime}, w^{\prime \prime}\right)$, such that $G$ still consists of quadrilaterals. By that modification, the degree of $v_{1}$ decreases by one, and we can continue by induction. Observe that neither $w^{\prime}$ nor $w^{\prime \prime}$ can be an internal vertex of $G$, as otherwise $G$ contains an internal quadrilateral, in contradiction to Lemma B.2, Case II.

Case IV: $\operatorname{degree}\left(v_{1}\right)>3$ and degree $\left(v_{2}\right)=3$, and no vertex incident to $v_{1}$ or $v_{2}$ is strictly convex relative to $C$.

Then $P=(3,3,2,2, \ldots, 2)$.

Case V: $\operatorname{degree}\left(v_{1}\right)=3$ and $\operatorname{degree}\left(v_{2}\right)=3$.

Then nothing is to show.

Note that the exception $P=(3,3,2,2, \ldots, 2)$ does not contradict Lemma 3.5 either; its (unique) optimal decomposition can be regarded as a 2-cut plus a perfect core polygon.

\section{B.3 Combinations of Internal Compo- nents}

Now we are in a position to restrict our attention essentially to the area components described in Lemma 3.5.
Lemma B.10 For any polygon $P \notin\{(3,3,3,3)$, $(2,1,1),(4,3,3),(4,2,2)\}$, there is a conformal decomposition $G$ with minimum $q(G)$ which has an area decomposition such that there is at most one outerplanar area component and all other area components are isomorphic to one of the cut components in Figs. 8 or 9. Furthermore, no two cut components share an edge in that area decomposition.

Proof: By Lemma B.6, there is an optimal decomposition that each connected component of $G^{i}$ falls into one of the following categories:

1. it is an isolated vertex,

2. it is an isolated edge, or

3. any two incident edges form a situation as depicted in Fig. 13 or 16.

By Lemmas B.8 and B.9, the vertices which fall into the first two categories can be assumed to have degree three. By Corollary B.7, all larger interior components induce a $(2 k, 1,1)$ with one trivial segment belonging to $P$.

Given such a decomposition, we have to find an area decomposition as claimed in the lemma.

If there is no interior component, the decomposition is perfect and we have just one single, outerplanar area component, namely $G$ itself.

So let us assume that $G$ has interior components. We give an algorithmic proof which builds the area decomposition by induction on the number of interior components. Start with an area decomposition which has only one area component $G^{\prime}:=G$.

If there is an interior component of more than two vertices, all quadrilaterals incident to this component together form a cut component of the type in Fig. 9, as we have seen above. Hence, we can take this area component away from $G^{\prime}$ and continue with one interior component less.

Next consider the case of an isolated internal vertex $v$. As $v$ has degree three, it is incident to exactly three quadrilaterals which form a cycle $C$ of length six. Note that $C$ contains an interval of length four which belongs to $P$, as otherwise the cycle $C$ can be refined without internal vertex which contradicts optimality. If two of the three 


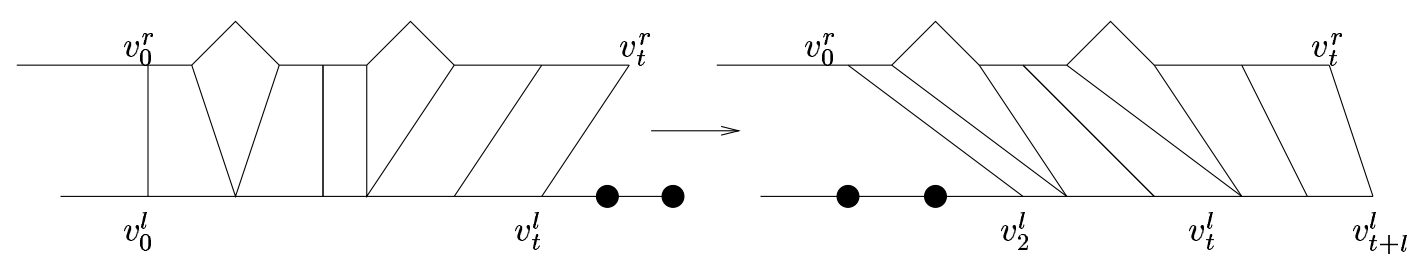

Figure 21: $(p, q)$-shift - here: a $(2,0)$-shift

quadrilaterals of $C$ are incident to two edges lying on $P$ they form a cut component of type (c) in Fig. 8. If only one quadrilateral is incident to two edges of $P$, we take this as a cut component of type (b). At least one quadrilateral must have the latter property, and so we can also reduce $G^{\prime}$ in this case.

Finally, consider an isolated internal edge $e=$ $\left(v_{1}, v_{2}\right)$. As both $v_{1}, v_{2}$ have degree three, they are incident to exactly four quadrilaterals which also form a cycle $C$ of length six. Exactly three consecutive edges of $C$ belong to the same segment of $P$ as otherwise the current decomposition of $C$ cannot be optimal. If two more edges of $C$ belong to $P$, we either form a cut component of type (d) or (e) in Fig. 8. Otherwise, the quadrilateral incident to the second edge of the three which belong to the same segment of $P$ is taken as an area component of type (a) in Fig. 8.

Note that the area component $G^{\prime}$ always remains connected if we take away one of the cut components in the above procedure. Note further that if we define the area components as we did, no two cut components can share an edge.

With these lemmata at hand it is not too difficult to prove the remaining statements of Lemma 3.5. The proof technique is as follows: We suppose for contradiction that there is no optimal conformal decomposition such that $G$ has the form according to Lemma 3.5. Since we know so far that there is an optimal conformal decomposition with an area decomposition into cut components plus at most one single core component, this means that we have applied too many cut operations, or equivalently, $G$ has too many internal components. Hence, we have to show that there is another optimal conformal decomposition $G^{\prime}$ which uses at least one internal component less, and then we are done by induction. The other pos- sibility is to derive a contradiction to optimality. This is achieved in a case analysis for the different kinds of operations. Roughly speaking, the idea is to "unite" two internal components to a single larger one (with not more internal vertices in total) and to rebuild the conformal decomposition afterwards.

The latter involves the "shifting" of a whole bunch of quadrilaterals of $G$ along some dual path. Let us first define what we mean by a $(p, q)$-shift applied to some shortest dual path in a conformal decomposition, see Fig. 21 for an intuitive understanding.

Let $G^{*}$ be the dual internal graph of some feasible conformal decomposition, and $V_{1}^{*}-V_{2}^{*}-\ldots-$ $V_{s-1}^{*}-V_{s}^{*}$ some shortest path in $G^{*}$ between $V_{1}^{*}$ and $V_{s}^{*}$. Corresponding to a dual path, there are two primal paths $p_{l}, p_{r}$, incident to these quadrilaterals on the dual path's "left-hand" or "righthand" side. A vertex of $p_{l}$ or $p_{r}$ is covered if it is an endvertex of an edge going from $p_{l}$ to $p_{r}$, and uncovered otherwise.

The shift consists of three steps:

1. Contract all those quadrilaterals which are incident to an uncovered vertex. That is, identify each uncovered vertex with its two neighbors on $p_{l}$ or $p_{r}$. As a result, we get paths $\tilde{p}_{l}$ and $\tilde{p}_{r}$ of the same length $t$.

2. Let $v_{0}^{l}, \ldots, v_{t}^{l}\left(v_{0}^{r}, \ldots, v_{t}^{r}\right)$ be the vertices of $\tilde{p}_{l}$ $\left(\tilde{p}_{r}\right)$. Assume further, that there is a path of $p$ (primal) edges starting at $v_{t}^{l}$, whose vertices $v_{t+1}^{l}, \ldots, v_{t+p}^{l}$ we want to cover. Similarly, there is a path of $r$ edges, starting at $v_{0}^{r}$, with vertices $v_{-1}^{r}, \ldots, v_{-q}^{r}$. In this step, the edges $e_{i}=\left(v_{i}^{l}, v_{i}^{r}\right)$ are replaced (i.e. "shifted") by edges $\tilde{e}_{i}=\left(v_{i+p}^{l}, v_{i-q}^{r}\right)$.

3. Reverse the contraction of the first step. 

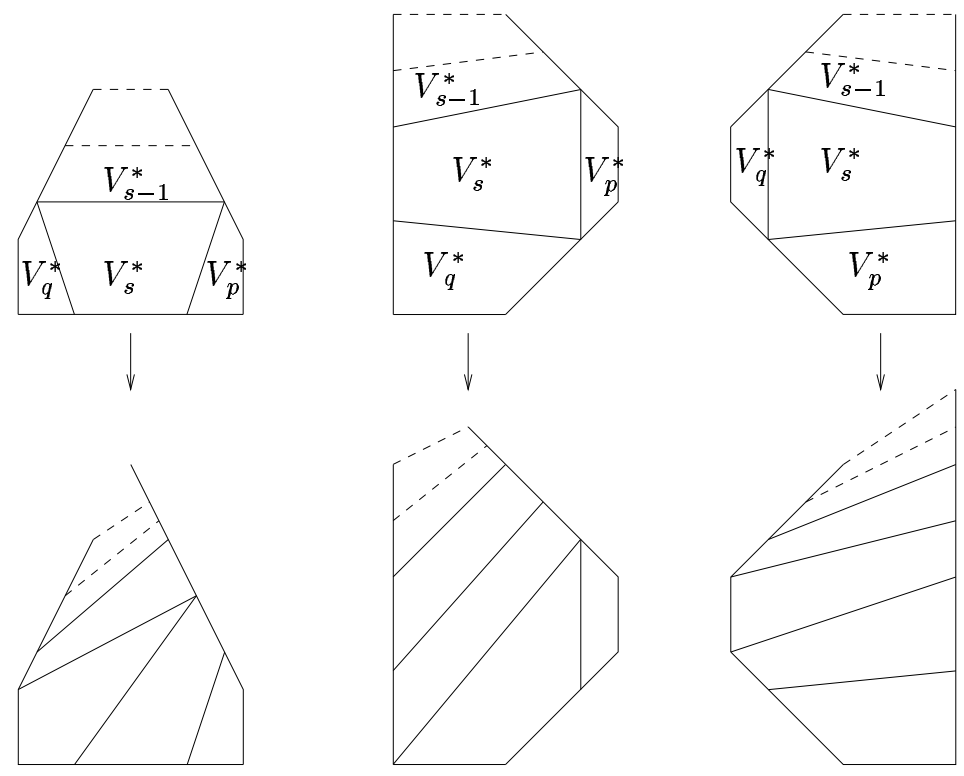

Figure 22: How to avoid $(2 k, 1,1)$-cuts in non path-solutions

The whole operation is called a $(p, q)-$ shift.

Lemma B.11 If a $(2 k, 1,1)$-cut appears in some optimal conformal decomposition, it is a leaf in a path-solution.

Proof: Suppose we have an optimal conformal decomposition $G$ of some polygon $P$ which involves some $(2 k, 1,1)$-cut.

Let $G_{t}^{*}$ be the graph obtained from the dual internal graph $G^{*}$ where all the vertices belonging to faces of the $(2 k, 1,1)$-cut are identified to $V_{t}$. We will show that there is no path $V_{t}^{*}-V_{2}^{*}-$ $\ldots-V_{s-1}^{*}-V_{s}^{*}$ in $G_{t}^{*}$, such that there are two other neighbors $V_{p}^{*}, V_{q}^{*}$ of $V_{s}^{*}$ which form an independent set with $V_{s-1}^{*}$. Having proved that, the lemma is immediate, since a $K_{1,3}$-like solution (or the $(2 k, 1,1)$-cut being no leaf) obviously implies such a vertex $V_{s}^{*}$ in its internal dual graph.

Hence, assume first that there is such a path starting at $V_{t}^{*}$, and take a shortest one. We want to show that there is a conformal decomposition which uses one internal vertex less than the current solution, which is a contradiction. We have to distinguish two cases: $k=2$ and $k \geq 3$. (Observe that a cut with $k=1$ will never appear in an optimal solution, unless $P=(2,1,1)$.)
Case $k \geq 3$ :

The idea is to replace the $(2 k, 1,1)$-cut be a $(2 k-2$,

1,1)-cut. By Lemma B.3, this saves exactly one internal vertex. The cut edge, however, changes its position and the last two vertices of the base segment of the $(2 k, 1,1)$-cut are not covered anymore. Take the base segment as the "left-handside" of the dual path. To cover them, we apply a $(2,0)$-shift to the path $V_{s-1}^{*}-\ldots-V_{2}^{*}$ (the path directed in this way), if this path is not empty. This then would give us two uncovered vertices incident to the former $V_{s-1}^{*}$.

Case $k=2$ :

If $k=2$, the $(4,1,1)$-cut is replaced by a 2 -cut. From the two trivial segments produced by the 2 cut, we select the one incident to the base segment of the $(4,1,1)$-cut as the cut edge. Again, we take the base segment as the "left-hand-side" of the dual path, but this time we apply a $(1,1)$-shift to the path $V_{s-1}^{*}-\ldots-V_{2}^{*}$.

In any case, we are now able to use the existence of $V_{s}^{*}$ and its independent neighbors $V_{p}^{*}, V_{q}^{*}$ to cover all vertices without introducing new internal vertices. The different cases (with the exception of symmetric ones) are illustrated in Fig. 22. 
Lemma B.12 (Restriction of cuts, reductions, and prolongations)

For $P \notin\{(2,1,1),(3,3,3,3),(4,3,3),(4,2,2)\}$, there is an optimal conformal decomposition of one of the following types:

a) the core polygon $P^{\prime}$ only admits a path solution, but not a $K_{1,3}$-like solution.

a1) If a leaf is cut, all applied operations yield cut edges, i.e. no 1-reduction or prolongation occurs. Furthermore, at most one $(2 k, 1,1)$-cut is used.

a2) If no leaf is cut, we may use a single 1reduction, or a single prolongation, but not more.

b) the core polygon $P^{\prime}$ admits a $K_{1,3}$-like solution. Cuts are only used to create the corresponding trivial segments, if necessary, and no other operation is applied elsewhere.

Proof: By Lemma B.10, there is an optimal conformal decomposition $G$ of $P$ which has an area decomposition into cut components and one perfect core component.

Suppose we have such an optimal conformal decomposition $G$ of $P$, as derived in the algorithmic proof of Lemma B.10 but $G$ has not the form of one of the above types as claimed in the lemma. Let $P^{\prime}$ be the surrounding cycle of the core component in the given area decomposition, henceforth called the core polygon.

Case a): The core polygon $P^{\prime}$ only admits a path-solution (but no $K_{1,3}$-like solution).

Let $s_{1}$ and $s_{2}$ be the trivial segments which are the leaves in the path-solution for $P^{\prime}$. If $s_{1}\left(s_{2}\right)$ is not an edge of $P$, let $G_{1}\left(G_{2}\right)$ be the cut component in the area decomposition incident to $s_{1}$ $\left(s_{2}\right)$. Denote by $P^{\prime \prime}$ the polygon which remains if we delete all quadrilaterals of $G_{1}$ and $G_{2}$ from $G$. Denote by $R_{1}$ and $R_{2}$ the intervals of $P^{\prime \prime}$ between $s_{1}$ and $s_{2}$. Assume further $L\left(R_{1}\right) \geq L\left(R_{2}\right)$. If this relation is fulfilled with equality, no further operation is necessary at all. Clearly, a prolongation has been applied to $R_{2}$, and a 1-reduction only to $R_{1}$.

Suppose that a 1-cut or a 2-cut has been applied which does not yield a leaf for $P^{\prime}$. We claim that this contradicts our assumption that $P^{\prime}$ does not admit a $K_{1,3}$-like solution). To see this, start with the area decomposition as defined in Lemma B.10. As all internal vertices incident to 1 -cuts and 2-cuts have degree three, there is exactly one quadrilateral incident to each such cut which belongs to the conformal decomposition $G_{P^{\prime}}$ of the core polygon $P^{\prime}$. If we remove all these quadrilaterals from $G_{P^{\prime}}$, the remaining graph is still outerplanar, and so admits either a path-solution or a $K_{1,3}$-like solution. In any case, if we add to such a solution the quadrilaterals which we just removed, this decomposition of $P^{\prime}$ cannot be a path.

Case a1): At least one leaf is a cut edge, and some other operation has been applied which does not yield the second leaf of the path.

Since a 1 -cut or a 2 -cut would allow a $K_{1,3^{-}}$ like solution, the type of this further operation can only be a 1-reduction or a prolongation.

The effect of a prolongation is to enlarge $L\left(R_{2}\right)$ by two units. As we get the same effect if we reduce $(L-2 K)\left(R_{1}\right)$ by two units, the idea is to use a "larger" cut instead of a prolongation. Hence, if we currently use a $1-$ cut, it will be replaced by a 2 -cut, a 2 -cut will be replaced by a $(4,1,1)$-cut, and a $(2 k, 1,1)$-cut can be replaced by a $(2 k+2,1,1)$-cut. Note that by the definition of a $(2 k, 1,1)$-cut, the base segment has to be a segment of the original polygon. Thus it might be impossible to apply a $(2 k, 1,1)$-cut if the corresponding segment is too short. But in these situations we can always use the strictly convex vertex which makes the base segment too short, and apply there a cheaper cut. We so still yield the desired effect on $(L-2 K)\left(R_{1}\right)$. In fact, a case analysis shows that the existence of a prolongation always leads to a contradiction to optimality.

A 1-reduction has the effect to shorten $(L-2 K)\left(R_{1}\right)$ by two units (or is useless). But the same idea as for prolongations works in this case: we replace the current cut in the same manner as above, and delete the 1-reduction which is closest on $R_{1}$ to the leaf. By that, we only change the place on $R_{1}$ where to shorten its length, but not $(L-2 K)\left(R_{1}\right)$ itself. A complete decomposition can be rebuilt using an appropriate shift. 
So far we have ruled out the use of 1-reductions or prolongations. It remains the case, that at both leaves a $(2 k, 1,1)$-cut has been applied.

Hence, suppose that an optimal conformal decomposition consists of some $\left(2 k_{1}, 1,1\right)$-cut and some $\left(2 k_{2}, 1,1\right)$-cut, with $k_{1} \geq k_{2}$, and that there is a unique dual path $V_{1}^{*}-\ldots-V_{s}^{*}$ of $s$ quadrilaterals between the two cut edges. It is easy to see that we may assume $s \geq 1$ (otherwise the decomposition cannot be optimal).

Let $v_{1}$ and $v_{2}$ be the two corners at which the cuts have been applied, and $I_{1}=I\left[v_{1}, v_{2}\right]$, $I_{2}=I\left[v_{2}, v_{1}\right]$ the corresponding intervals in $P$. If one base segment lies on $I_{1}$ and the other on $I_{2}$, the current solution cannot be optimal, as we may replace both $\left(2 k_{i}, 1,1\right)$-cuts by 2 -cuts at the same corners $v_{1}$ and $v_{2}$ and rebuild a feasible decomposition by applying a $(2,2)$-shift to $V_{1}^{*}-\ldots-V_{s}^{*}$.

Hence, we may assume that both base segments belong to $I_{1}$.

If the base segment, say $S_{1}$, of the $\left(2 k_{1}, 1,1\right)-$ cut has length $L\left(S_{1}\right) \geq 2 k_{1}+3$, we may replace the $\left(2 k_{1}, 1,1\right)$-cut by a $\left(2 k_{1}+2,1,1\right)$-cut. If the base segment $S_{1}$ has only length $L\left(S_{1}\right) \leq 2 k_{2}+2$, we still use a $\left(2 k_{1}, 1,1\right)$-cut, but at the other corner of $S_{1}$.

In any case, we replace the other $\left(2 k_{2}, 1,1\right)$-cut by a 2 -cut, if $k_{2}=2$, or by a $\left(2 k_{2}-2,1,1\right)$ cut, otherwise, and can rebuild a conformal decomposition by an appropriate shift. Clearly, we can continue in the same way, until the second $\left(2 k_{2}, 1,1\right)$-cut vanishes.

This finishes the case of two $(2 k, 1,1)$-cuts as leaves of a path-solution.

Case a2): If no leaf is a cut edge, but a prolongation and one other operation (i.e., another prolongation or a 1-reduction) has been applied, these use together at least 3 internal vertices. Hence, we may also use a $(4,1,1)$-cut at the former leaf to yield the same effect.

It remains the case that no leaf is a cut edge, but more than one 1-reduction has been used. The idea is then to replace two 1-reductions by a 2 -cut which is based at the strictly convex vertex of one of the 1-reductions. Let us choose two 1reductions where the interval between them on $R_{1}$ does not contain another 1-reduction. Apply the 2-cut instead of one of them and take as a cut edge the trivial segment closer on $R_{1}$ to the other 1 reduction. Observe that we may assume because of Lemma B. 8 that the internal vertices belonging to the 1-reductions all have degree 3 , and that one of the three edges of an internal vertex is incident to $R_{2}$. In particular, this observation applies to the chosen second 1-reduction. The corresponding vertex incident to $R_{2}$, say $v_{3}$, must be strictly convex relative to the subpolygon formed by all quadrilaterals of the path solution between the two 1-reductions. The two other vertices lying on $R_{1}$, say $v_{1}, v_{2}$, have to be covered when we delete the second 1-reduction from the current solution. Both can be covered by the first neighbor of $v_{3}$ in direction to the new 2-cut. The rest of the subpolygon is then perfect. Note that we can do this modification in such a way that for no internal vertex which belongs to some other operation the degree changes. Hence, we can continue by induction.

Case b): The current core polygon $P^{\prime}$ has a $K_{1,3^{-}}$ like solution, and some operation is applied, which does not create one of the leaves.

We want to show that such a situation contradicts optimality. Denote by $s_{1}, s_{2}, s_{3}$ the trivial segments which form the leaves of the current solution, and $R_{1}$ the interval between $s_{1}$ and $s_{2}, R_{2}$ the interval between $s_{2}$ and $s_{3}, R_{3}$ the interval between $s_{3}$ and $s_{1}$, the intervals $R_{i}$ taken with respect to the core polygon $P^{\prime}$. Assume further, that the quadrilateral $V_{C}$ corresponding to the dual vertex of degree 3 in the core polygon $P^{\prime}$ has an edge $\left(v_{1}, v_{2}\right)$ incident to $R_{1}$.

The applied operation cannot be a $(2 k, 1,1)-$ cut, as we have seen in Lemma B.11. If there is a 2-cut with an effective reduction of 4 units, we may instead apply a 1-reduction at the same strictly convex vertex which has only an effective reduction of 2 units. All other types of reductions and cuts have an effective reduction of 2 units. Hence, if we undo one such operation or do the replacement to the 1-reduction in the other case, we get exactly 2 uncovered vertices.

Suppose first, that the two uncovered vertices lie on $R_{1}$. We may assume that the two uncovered vertices are directly neighbored to $v_{1}, v_{2}$ by using an appropriate $(k, 0)$-shift, but delay the last re- 

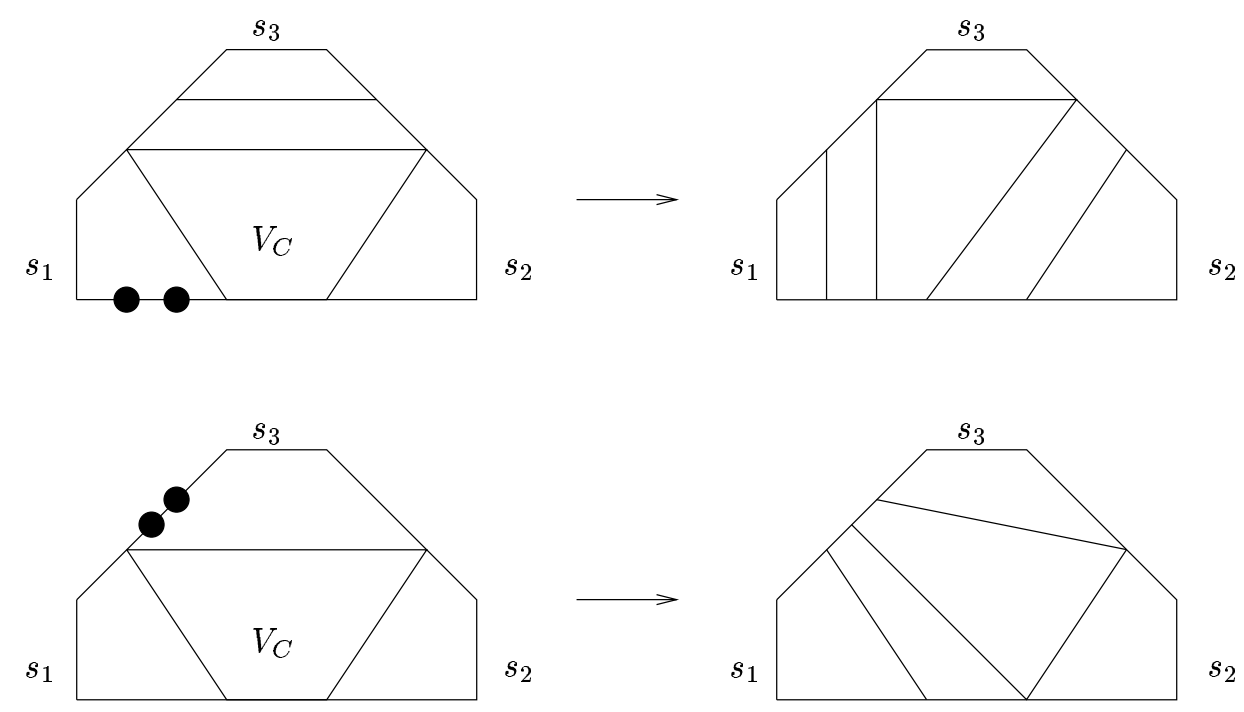

Figure 23: Sketch: How to avoid cuts and reductions in $K_{1,3}$-like solutions

versing step of the shift. Using the quadrilateral adjacent to $V_{C}$ on the path leading to $s_{3}$, the center $V_{C}$ itself and the two uncovered vertices, we can find a perfect conformal decomposition of the induced $(3,1,1,1,1,1)$, see a sketch in Fig. 23. (Note that this transformation changes the $K_{1,3^{-}}$ like solution to a path solution if the path from $V_{C}$ to $s_{3}$ contains only a single quadrilateral.) Finally, we rebuild those parts which have been shrunken away in the shift.

Suppose now, that the two uncovered vertices lie on $R_{2}$ (or on $R_{3}$, the symmetric case). Again we may assume that the two uncovered vertices are directly neighbored to $V_{C}$ by using an appropriate $(k, 0)$-shift without the last reversing step. In this case we take only $V_{C}$ and the two uncovered vertices which together induce a $(2,1,1,1,1)$. Clearly, the latter polygon is perfect. The last step is again the rebuilding of shrunken quadrilaterals.

It remains the case that a prolongation has been applied. But observe that the effect of a prolongation is just the opposite one of an reduction or cut. Thus by reversing the above transformations for cuts and reductions, we easily get improved solutions.

\section{Proof of Theorem 4.1}

Proof: Exact Cover by 3-Sets is the following problem. Given a ground set $U=\left\{u_{1}, \ldots, u_{3 m}\right\}$ and a family $S_{1}, S_{2}, \ldots, S_{n}$ of subsets of $U$, each of cardinality three, is there a subfamily of $m$ subsets that covers $U$ ? This problem is well-known to be strongly $\mathcal{N} \mathcal{P}$-complete [3]. The reduction from Exact Cover by 3-Sets to the minimum conformal mesh refinement problem is as follows.

For each subset $S_{i}$ we create a polygon as in Fig. 24. For simplicity we will refer to it as polygon $S_{i}$. Edge $e_{l S_{i}}$ with $l \in\{1,2,3\}$ is to correspond with the $l$-th element of $S_{i}$. If an edge $e_{l S_{i}}$ is subdivided this will mean that subset $S_{i}$ covers the corresponding element.

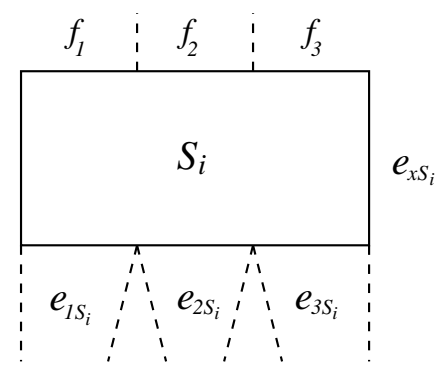

Figure 24: Polygon for a subset $S_{i}$.

Suppose that the edges $f_{1}, f_{2}, f_{3}$ cannot be subdivided in any optimal solution. This can be as- 
sumed by the following construction. Add a homogeneous bunch of conforming quadrilaterals to such an edge $e$ such that the shortest path from it to the mesh boundary has a length of at least $k$, where $k$ is some constant to be specified later. Each bunch needs at most $O\left(k^{2}\right)$ quadrilaterals. By parity, the subdivision of $f_{i}$ by one unit would imply at least $k$ additional quadrilaterals in any feasible solution. If no edge of $S_{i}$ is subdivided at all, then we clearly need exactly 3 quadrilaterals. If one subdivides each of the edges $e_{l S_{i}}$ and $x_{S_{i}}$ exactly once, then we need exactly 7 quadrilaterals. If only one edge $e_{l S_{i}}$ is subdivided once, then $x_{S_{i}}$ must be subdivided by parity reasons, and we need 5 quadrilaterals. If exactly two edges $e_{l S_{i}}$ are subdivided once, one needs exactly 6 quadrilaterals.

Hence, in an exact cover one needs exactly $7 m+3(n-m)=3 n+4 m$ quadrilaterals for all subsets $S_{i}$ together. If an exact cover exists, it is not worth to cover three times only one element, as this needs 15 quadrilaterals compared to $7+2 \cdot 3=13$. Furthermore, it is more expensive to cover once only one element and once two elements (and once no element), as this needs $5+6+3=14$ quadrilaterals. Finally, it is more expensive to cover three times two elements, as this needs 18 quadrilaterals compared with 17 quadrilaterals in an exact cover. If no exact cover exists one needs more than $3 n+4 m$ quadrilaterals for the refinement of all subsets $S_{i}$.

Let $k_{i}$ denote the total number of appearances of $u_{i}$ in the subsets $S_{j}$. Define for each element $u_{i}$ a polygon as in Fig. 25.

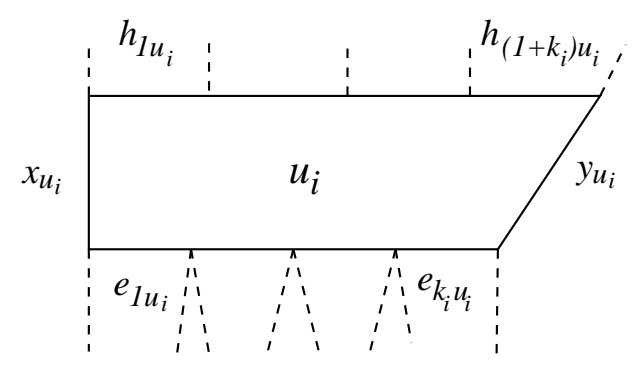

Figure 25: Polygon for an element $u_{i}$.

The parity of such an "element" polygon $u_{i}$ is odd. Suppose that none of its edges $h_{1 u_{i}}, \ldots, h_{k_{i} u_{i}}, h_{\left(1+k_{i}\right) u_{i}}, x_{i u_{i}}, y_{i u_{i}}$ is subdivided. This can be achieved by the same trick with additional quadrilaterals as above. Then a conformal refinement of $u_{i}$ requires that an odd number of additional points is placed on the edges $e_{1 u_{i}}, \ldots, e_{k_{i} u_{i}}$. In particular, the optimum refinement for $u_{i}$ is achieved if we add exactly one additional point on one of these edges. In this case, $u_{i}$ becomes perfect and needs exactly $1+k_{i}$ quadrilaterals.

Now we establish the relationship between elements $u_{i}$ and subsets $S_{j}$. If $u_{i}$ is an element of $S_{j}$, we connect an edge $e_{l S_{j}}$ with a corresponding edge $e_{r u_{i}}$ by a chain of $q$ conforming quadrilaterals ( $q$ is some small constant) as in Fig. 26 which we call channels.

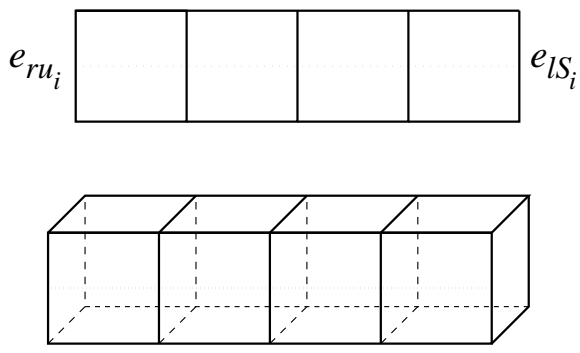

Figure 26: "Channel" between element $u_{i}$ and subset $S_{i}$ before and after blowing up. The pointed line denotes the split if this channel is used.

Clearly, all these channels can easily be embedded into the three-dimensional space such that they do not cross each other. The purpose of these connections is to make sure that each element $u_{i}$ may be covered by any subset $S_{j}$ it belongs to. All channels have the same length such that it makes no difference which one is chosen to cover an element.

We also want that if $e_{r u_{i}}$ is subdivided once then also $e_{l S_{j}}$ is subdivided once in any optimal solution. Hence, it must be cheaper to split each quadrilateral on the channel into two quadrilaterals than to use any other feasible refinement. To this end, we "blow up" each channel: we take four copies of each channel and glue them together as in Fig. 26. As all these quadrilaterals are conforming, a subdivision by one unit of the original edge $e_{r u_{i}}$ implies by parity arguments an odd subdivision of either the original edge $e_{l S_{j}}$ as intended or of one of the copies of $e_{r u_{i}}$ or $e_{l S_{j}}$. In the first case, the original path is split into two chains. To 
rule out the latter case, we have to make such a subdivision expensive. This can be done by the same trick with additional bunches of conforming quadrilaterals as above. If the constant $k$ is chosen larger than $q$, then it is easy to see that any optimal solution uses exactly one channel per element $u_{i}$. From the above discussion it is also clear that an exact cover is cheaper than any other cover of $U$.

Observe that no folding edge occurs in our construction. Hence, the theorem remains true in the special case of homogeneous meshes.

\section{Detailed Proof of Theo- rem 4.2}

Proof: It remains to show

$$
\min \left(P_{X}\right) \leq 2 \cdot \operatorname{Min}(P)+\left|E_{P}\right|-2
$$

for $X \equiv 1$. Note that all segments of $P_{X}$ have even lengths. Hence, $P_{X}$ is different from all polygons listed in Lemma 3.5 except possibly for $(4,2,2)$. Ineq. (1) is easy to check by hand for $P_{X}=(4,2,2)$, which means $P=(2,1,1)$. Therefore, in the rest of this proof we assume that $P_{X}$ is none of the polygons listed in Lemma 3.5.

Let $i(X)$ denote the number of internal vertices in an optimal conformal decomposition of $P_{X}$. The proof of Lemma 3.2 yields that this value is the same for all optimal conformal decompositions, namely

$$
\begin{aligned}
\min \left(P_{X}\right) & =i(X)+\frac{\left|E_{P_{X}}\right|}{2}-1 \\
& =i(X)+\left|E_{P}\right|-1 .
\end{aligned}
$$

Hence, it suffices to show

$$
i(X) \leq 2 \cdot \operatorname{Min}(P)-1 .
$$

The proof of Lemma 3.2 also yields

$$
\operatorname{Min}(P) \geq \frac{\left|E_{P}\right|}{2}-1 .
$$

Therefore, Ineq. (1) is shown if

$$
i(X) \leq\left|E_{P}\right|-3 .
$$

Since $P_{X}$ is none of the polygons listed in Lemma 3.5, there is an optimal decomposition for $P_{X}$ that can be partitioned into one outerplanar area component $G^{0}$ and cut components $G^{j}$ with $j=1, \ldots, \ell, \ell \leq 3$. For $j=0, \ldots, \ell$, let $E^{j}$ denote the edges of $P_{X}$ belonging to $G^{j}$. For $j=1, \ldots, \ell$, let $i(j, X)$ denote the number of internal vertices introduced by the cut component $G^{i}$. For a cut component $G^{j}$ of type (b) or (c) in Fig. 8, this means $i(j, X)=1$; for the other types in Fig. 8 this means $i(j, X)=2$; and for a cut component of the type in Fig. 9 with $2 k+1$ edges this means $i(j, X)=k+1$. Clearly,

$$
\left|E_{P}\right|=\sum_{j=0}^{\ell}\left|E^{j}\right|,
$$

and

$$
i(X)=\sum_{j=1}^{\ell} i(j, X)
$$

Obviously, we have $\left|E^{j}\right|-i(j, X) \geq 2$ for each $j=1, \ldots, k$. Hence, if the optimal conformal decomposition of $P_{X}$ contains two or more cut components, Ineq. (5) is immediate. Otherwise, the core component contains a leaf that is a trivial segment of $P_{X}$. This means $\left|E^{0}\right| \geq 1$, and Ineq. (5) is immediate again. 